\title{
Modified noor iterations for nonexpansive semigroups with generalized contraction in Banach spaces
}

\author{
Rabian Wangkeeree ${ }^{1,2^{*}}$ and Pakkapon Preechasilp ${ }^{1}$
}

* Correspondence: rabianw@nu.ac.

'Department of Mathematics, Faculty of Science, Naresuan University, Phitsanulok 65000, Thailand

Full list of author information is available at the end of the article

\begin{abstract}
In this article, the modified Noor iterations are considered for the generalized contraction and a nonexpansive semigroup in the framework of a reflexive Banach space which admits a weakly sequentially continuous duality mapping. The strong convergence theorems are obtained under very mild conditions imposed the parameters. The results presented in this article improve and extend the corresponding results announced by Chen and He and Chen et al. and many others. AMS subject classification: 47H09; 47H10; 47H17.

Keywords: generalized contraction, Meir-Keeler type mapping, nonexpansive semigroup, fixed point, reflexive Banach space
\end{abstract}

\section{Introduction and preliminaries}

Let $E$ be a real Banach space. A mapping $T$ of $E$ into itself is said to be nonexpansive if $\|T x-T y\| \leq\|x-y\|$ for each $x, y \in E$. We denote by $\operatorname{Fix}(T)$ the set of fixed points of T. A mapping $f: E \rightarrow E$ is called $\alpha$-contraction, if there exists a constant $0<\alpha<1$ such that $\|f(x)-f(y)\| \leq \alpha|| x-y||$ for all $x, y \in E$. Throughout this article, we denote by $\mathbb{N}$ and $\mathbb{R}^{+}$the sets of positive integers and nonnegative real numbers, respectively. A mapping $\psi: \mathbb{R}^{+} \rightarrow \mathbb{R}^{+}$is said to be an L-function if $\psi(0)=0, \psi(t)>0$, for each $t>0$ and for every $t>0$ and for every $s>0$ there exists $u>s$ such that $\psi(t) \leq s$, for all $t \in[s, u]$, As a consequence, every $L$-function $\psi$ satisfies $\psi(t)<t$, for each $t>0$.

Definition 1.1. Let $(X, d)$ be a matric space. A mapping $f: X \rightarrow X$ is said to be :

(i) a $(\psi, L)$-function if $\psi: \mathbb{R}^{+} \rightarrow \mathbb{R}^{+}$is an $L$-function and $d(f(x), f(y))<\psi(d(x, y))$, for all $x, y \in X$, with $x \neq y$ :

(ii) a Meir-Keeler type mapping if for each $\varepsilon>0$ there exists $\delta=\delta(\varepsilon)>0$ such that for each $x, y \in X$, with $\varepsilon \leq d(x, y)<\varepsilon+\delta$ we have $d(f(x), f(y))<\varepsilon$.

If, in Definition 1.1 we consider $\psi(t)=\alpha t$, for each $t \in \mathbb{R}^{+}$, where $\alpha \in[0,1)$, then we get the usual contraction mapping with coefficient $\alpha$.

Proposition 1.2. [1] Let $(X, d)$ be a matric space and $f: X \rightarrow X$ be a mapping. The following assertions are equivalent:

(i) $f$ is a Meir-Keeler type mapping :

(ii) there exists an L-function $\psi: \mathbb{R}^{+} \rightarrow \mathbb{R}^{+}$such that $f$ is a $(\psi, L)$-contraction.

(C) 2012 Wangkeeree and Preechasilp; licensee Springer. This is an Open Access article distributed under the terms of the Creative Commons Attribution License (http://creativecommons.org/licenses/by/2.0), which permits unrestricted use, distribution, and reproduction in any medium, provided the original work is properly cited. 
Lemma 1.3. [2] Let $X$ be a Banach space and $C$ be a convex subset of it. Let $T: C \rightarrow C$ be a nonexpansive mapping and $f$ is a $(\psi, L)$-contraction. Then the following assertions hold:

(i) $T$ o $f$ is a $(\psi, L)$-contraction on $C$ and has a unique fixed point in $C$;

(ii) for each $\alpha \in(0,1)$ the mapping $x \mapsto \alpha f(x)+(1-\alpha) T(x)$ is a Meir-Keeler type mapping on $C$.

Lemma 1.4. [3, Proposition 2] Let E be a Banach space and $C$ a convex subset of it. Let $f: C \rightarrow C$ be a Meir-Keeler type mapping. Then for each $\varepsilon>0$ there exists $r \in(0,1)$ such that for each $x, y \in C$ with $\|x-y\| \geq \varepsilon$ we have $\|f(x)-f(y)\| \leq r\|x-y\|$.

From now on, by a generalized contraction mapping we mean a Meir-Keeler type mapping or $(\psi, L)$-contraction. In the rest of the article we suppose that the $\psi$ from the definition of the $(\psi, L)$-contraction is continuous, strictly increasing and $\eta(t)$ is strictly increasing and onto, where $\eta(t):=t-\psi(t), t \in \mathbb{R}^{+}$. As a consequence, we have the $\eta$ is a bijection on $\mathbb{R}^{+}$.

A family $\mathcal{S}=\{T(t): 0 \leq t<\infty\}$ of mappings of $E$ into itself is called a nonexpansive semigroup on $E$ if it satisfies the following conditions:

(i) $T(0) x=x$ for all $x \in E$;

(ii) $T(s+t)=T(s) T(t)$ for all $s, t>0$;

(iii) $\|T(t) x-T(t) y\| \leq\|x-y\|$ for all $x, y \in E$ and $t \geq 0$;

(iv) for all $x \in E$, the mapping $t \mapsto T(t) x$ is continuous.

We denote by $\operatorname{Fix}(\mathcal{S})$ the set of all common fixed points of $\mathcal{S}$, that is,

$$
\operatorname{Fix}(\mathcal{S}):=\{x \in E: T(t) x=x, \quad 0 \leq t<\infty\}=\cap_{t \geq 0} \operatorname{Fix}(T(t)) .
$$

In [4], Shioji and Takahashi introduced the following implicit iteration in a Hilbert space

$$
x_{n}=\alpha_{n} x+\left(1-\alpha_{n}\right) \frac{1}{t_{n}} \int_{0}^{t_{n}} T(s) x_{n} d s, \quad \forall n \in \mathbb{N}
$$

where $\left\{\alpha_{n}\right\}$ is a sequence in $(0,1),\left\{t_{n}\right\}$ is a sequence of positive real numbers which diverges to $\infty$. Under certain restrictions on the sequence $\left\{\alpha_{n}\right\}$, Shioji and Takahashi [4] proved strong convergence of the sequence $\left\{x_{n}\right\}$ to a member of $F(\mathcal{S})$. In [5], Shimizu and Takahashi studied the strong convergence of the sequence $\left\{x_{n}\right\}$ defined by

$$
x_{n+1}=\alpha_{n} x+\left(1-\alpha_{n}\right) \frac{1}{t_{n}} \int_{0}^{t_{n}} T(s) x_{n} d s, \quad \forall n \in \mathbb{N}
$$

in a real Hilbert space where $\{T(t): t \geq 0\}$ is a strongly continuous semigroup of nonexpansive mappings on a closed convex subset $C$ of a Banach space $E$ and $\lim _{n \rightarrow \infty}$ $t_{n}=\infty$. Using viscosity iterative method, Chen and Song [6] studied the strong convergence of the following iterative method for a nonexpansive semigroup $\{T(t): t \geq 0\}$ with $\operatorname{Fix}(\mathcal{S}) \neq \emptyset$ in a Banach space: 


$$
x_{n+1}=\alpha_{n} f(x)+\left(1-\alpha_{n}\right) \frac{1}{t_{n}} \int_{0}^{t_{n}} T(s) x_{n} d s, \quad \forall n \in \mathbb{N},
$$

where $f$ is a contraction. Note however that their iterate $x_{n}$ at step $n$ is constructed through the average of the semigroup over the interval $(0, t)$. Suzuki [7] was the first to introduce again in a Hilbert space the following implicit iteration process:

$$
x_{n}=\alpha_{n} u+\left(1-\alpha_{n}\right) T\left(t_{n}\right) x_{n}, \quad \forall n \in \mathbb{N},
$$

for the nonexpansive semigroup case. In 2002, Benavides et al. [8] in a uniformly smooth Banach space, showed that if $\mathcal{S}$ satisfies an asymptotic regularity condition and $\left\{\alpha_{n}\right\}$ fulfills the control conditions $\lim _{n \rightarrow \infty} \alpha_{n}=0, \sum_{n=1}^{\infty} \alpha_{n}=\infty$, and $\lim _{n \rightarrow \infty} \frac{\alpha_{n}}{\alpha_{n+1}}=0$, then both the implicit iteration process (1.4) and the explicit iteration process (1.5)

$$
x_{n+1}=\alpha_{n} u+\left(1-\alpha_{n}\right) T\left(t_{n}\right) x_{n}, \quad \forall n \in \mathbb{N},
$$

converge to a same point of $F(\mathcal{S})$. In 2005, Xu [9] studied the strong convergence of the implicit iteration process (1.1) and (1.4) in a uniformly convex Banach space which admits a weakly sequentially continuous duality mapping. Recently Chen and He [10] introduced the viscosity approximation methods:

$$
y_{n}=\alpha_{n} f\left(y_{n}\right)+\left(1-\alpha_{n}\right) T\left(t_{n}\right) y_{n}, \quad \forall n \in \mathbb{N},
$$

and

$$
x_{n+1}=\alpha_{n} f\left(x_{n}\right)+\left(1-\alpha_{n}\right) T\left(t_{n}\right) x_{n}, \quad \forall n \in \mathbb{N},
$$

where $f$ is a contraction, $\left\{\alpha_{n}\right\}$ is a sequence in $(0,1)$ and a nonexpansive semigroup $\{T$ $(t): t \geq 0\}$. The strong convergence theorem of $\left\{x_{n}\right\}$ is proved in a reflexive Banach space which admits a weakly sequentially continuous duality mapping. Very recently, motivated by the above results, Chen et al. [11] proposed the following two modified Mann iterations for nonexpansive semigroups $\{T(t): 0 \leq t<\infty\}$ and obtained the strong convergence theorems in a reflexive Banach space $E$ which admits a weakly sequentially continuous duality mapping:

$$
\left\{\begin{array}{c}
y_{n}=\alpha_{n} x_{n}+\left(1-\alpha_{n}\right) T\left(t_{n}\right) x_{n} \\
x_{n}=\beta_{n} f\left(x_{n}\right)+\left(1-\beta_{n}\right) y_{n}
\end{array}\right.
$$

and

$$
\left\{\begin{array}{l}
x_{0} \in C \\
y_{n}=\alpha_{n} x_{n}+\left(1-\alpha_{n}\right) T\left(t_{n}\right) x_{n} \\
x_{n+1}=\beta_{n} f\left(x_{n}\right)+\left(1-\beta_{n}\right) y_{n}
\end{array}\right.
$$

where $f: C \rightarrow C$ is a contraction. They proved that the implicit iterative scheme $\left\{x_{n}\right\}$ defined by (1.8) converges to an element $q$ of $\operatorname{Fix}(\mathcal{S})$, which solves the following variation inequality problem:

$$
\langle(f-I) q, j(x-q)\rangle \leq 0 \quad \text { for all } x \in \operatorname{Fix}(\mathcal{S}) .
$$

Furthermore, Moudafi's viscosity approximation methods have been recently studies by many authors; see the well known results in $[12,13]$. However, the involved mapping $f$ is usually considered as a contraction. Note that Suzuki [14] proved the 
equivalence between Moudafi's viscosity approximation with contractions and Browder-type iterative processes (Halpern-type iterative processes); see [14] for more details.

In this article, inspired by above result, we introduce and study the explicit viscosity iterative scheme for the generalized contraction $f$ and a nonexpansive semigroup $\{T(t)$ : $t \geq 0\}$ :

$$
\left\{\begin{array}{l}
x_{0} \in C^{\prime} \\
z_{n}=\gamma_{n} x_{n}+\left(1-\gamma_{n}\right) T\left(t_{n}\right) x_{n} \\
y_{n}=\alpha_{n} x_{n}+\left(1-\alpha_{n}\right) T\left(t_{n}\right) z_{n} \\
x_{n+1}=\beta_{n} f\left(x_{n}\right)+\left(1-\beta_{n}\right) y_{n,} \quad n \geq 0 .
\end{array}\right.
$$

The iterative schemes (1.10) are called the three-step(modified Noor) iterations which inspired by three-step(Noor) iterations [15-23]. It is well known that three-step (Noor) iterations, include Mann and two-step iterative methods as special cases. If $\gamma \equiv$ 1, then (1.10) reduces to (1.9). Furthermore, the implicit iteration (1.8) and explicit iteration (1.10) are considered for the generalized contraction and a nonexpansive semigroup in the framework of a reflexive Banach space which admits a weakly sequentially continuous duality mapping. The strong convergence theorems are obtained under very mild conditions imposed the parameters. The results presented in this article improve and extend the corresponding results announced by Chen and $\mathrm{He}$ [10] and Chen et al. [11] and many others.

In order to prove our main results, we need the following lemmas.

Definition 1.5. [24] A Banach space is said to admit a weakly sequentially continuous normalized duality mapping $J$ from $E$ in $E^{*}$, if $J: E \rightarrow E^{*}$ is single-valued and weak to weak* sequentially continuous, that is, if $x_{n} \rightarrow x$ in $E$, then $J\left(x_{n}\right) \rightarrow^{*} J(x)$ in $E^{*}$.

A Banach space $E$ is said to satisfy Opial's condition if for any sequence $\left\{x_{n}\right\}$ in $E, x_{n}$ $\rightarrow x(n \rightarrow \infty)$ implies

$$
\limsup _{n \rightarrow \infty}\left\|x_{n}-x\right\|<\limsup _{n \rightarrow \infty}\left\|x_{n}-y\right\|, \quad \forall y \in E \text { with } x \neq y .
$$

By [25, Theorem 1], it is well known that if $E$ admits a weakly sequentially continuous duality mapping, then $E$ satisfies Opial's condition, and $E$ is smooth.

In order to prove our main result, we need the following lemmas.

Lemma 1.6. Let $E$ be a Banach space and $x, y \in E, j(x) \in J(x), j(x+y) \in J(x+y)$. Then

$$
\|x\|^{2}+2\langle y, j(x)\rangle \leq\|x+y\|^{2} \leq\|x\|^{2}+2\langle y, j(x+y)\rangle .
$$

In the following, we also need the following lemma that can be found in the existing literature [13,26].

Lemma 1.7. Let $\left\{a_{n}\right\}$ be a sequence of non-negative real numbers satisfying the property

$$
a_{n+1} \leq\left(1-\gamma_{n}\right) a_{n}+\delta_{n}, \quad n \geq 0,
$$

where $\left\{\gamma_{n}\right\} \subseteq(0,1)$ and $\left\{\delta_{n}\right\} \subseteq \mathbb{R}$ such that

$$
\sum_{n=1}^{\infty} \gamma_{n}=\infty \text {, and either } \lim \sup _{n \rightarrow \infty} \frac{\delta_{n}}{\gamma_{n}} \leq 0 \quad \text { or } \quad \sum_{n=1}^{\infty}\left|\delta_{n}\right|<\infty .
$$

Then $\lim _{n \rightarrow \infty} a_{n}=0$. 
Lemma 1.8. [27]Let $\left\{x_{n}\right\}$ and $\left\{y_{n}\right\}$ be bounded sequences in a Banach space E and $\left\{\beta_{n}\right\}$ a sequence in $[0,1]$ with $0<\lim _{\inf _{n \rightarrow \infty}} \beta_{n} \leq \lim \sup _{n \rightarrow \infty} \beta_{n}<1$. Suppose that $x_{n+1}$ $=\left(1-\beta_{n}\right) y_{n}+\beta_{n} x_{n}$ for all $n \geq 0$ and

$$
\limsup _{n \rightarrow \infty}\left(\left\|y_{n+1}-y_{n}\right\|-\left\|x_{n+1}-x_{n}\right\|\right) \leq 0 .
$$

Then $\lim _{n \rightarrow \infty}|| y_{n}-x_{n} \|=0$.

\section{Modified Mann iteration for generalized contractions}

Now, we are a position to state and prove our main results.

Theorem 2.1. Let E be a reflexive Banach space which admits a weakly sequenctially continuous duality mapping $J$ from $E$ into $E^{*}$, suppose $C$ is a nonempty closed convex subset of $E$. Let $\mathcal{S}:=\{T(t): t \geq 0\}$ be a nonexpansive semigroup on $C$ such that $\operatorname{Fix}(\mathcal{S}) \neq \emptyset$, and $f: C \rightarrow C$ a generalized contraction on $C$. Let $\left\{\alpha_{n}\right\} \subset(0,1),\left\{\beta_{n}\right\} \subset(0,1)$, and $\left\{t_{n}\right\} \subset(0, \infty)$ be sequences of real numbers satisfying $\lim _{n \rightarrow \infty} \alpha_{n}=\lim _{n \rightarrow \infty} t_{n}=\lim _{n \rightarrow \infty} \frac{\beta_{n}}{t_{n}}=0$. Define a sequence $\left\{x_{n}\right\}$ in $C$ by

$$
\left\{\begin{array}{l}
y_{n}=\alpha_{n} x_{n}+\left(1-\alpha_{n}\right) T\left(t_{n}\right) x_{n} \\
x_{n}=\beta_{n} f\left(x_{n}\right)+\left(1-\beta_{n}\right) y_{n}, \quad \text { for all } n \geq 1
\end{array}\right.
$$

Then $\left\{x_{n}\right\}$ converges strongly to $q$, as $n \rightarrow \infty ; q$ is the element of $\operatorname{Fix}(\mathcal{S})$ such that $q$ is the unique solution in $\operatorname{Fix}(\mathcal{S})$ to the following variational inequality:

$$
\langle(f-I) q, j(x-q)\rangle \leq 0 \quad \text { for all } x \in \operatorname{Fix}(\mathcal{S}) .
$$

Proof. We first show that $\left\{x_{n}\right\}$ is well defined. For any $n \geq 1$, we consider a mapping $\mathcal{G}_{n}$ on $C$ defined by

$$
\mathcal{G}_{n} x=\beta_{n} f(x)+\left(1-\beta_{n}\right) U_{n} x, \quad \forall x \in C,
$$

where $U_{n}:=\alpha_{n} I+\left(1-\alpha_{n}\right) T\left(t_{n}\right)$. It follows from nonexpansivity of $U_{n}$ and Lemma 1.3 that $\mathcal{G}_{n}$ is a Meir-Keeler type contraction. Hence $\mathcal{G}_{n}$ has a unique fixed point, denoted as $x_{n}$, which uniquely solves the fixed point equation

$$
x_{n}=\alpha_{n} f\left(x_{n}\right)+\left(1-\alpha_{n}\right) U_{n} x_{n}, \quad \forall n \geq 1 .
$$

Hence $\left\{x_{n}\right\}$ generated in (2.1) is well defined. Now we show that $\left\{x_{n}\right\}$ is bounded. Indeed, if we take a fixed point $x \in \operatorname{Fix}(\mathcal{S})$, we have

$$
\left\|y_{n}-x\right\| \leq \alpha_{n}\left\|x_{n}-x\right\|+\left(1-\alpha_{n}\right)\left\|T\left(t_{n}\right) x_{n}-x\right\| \leq\left\|x_{n}-x\right\|,
$$

and so

$$
\begin{aligned}
\left\|x_{n}-x\right\|^{2} & =\left\langle\beta_{n}\left(f\left(x_{n}\right)-x\right)+\left(1-\beta_{n}\right)\left(y_{n}-x\right), j\left(x_{n}-x\right)\right\rangle \\
& =\beta_{n}\left\langle f\left(x_{n}\right)-f(x)+f(x)-x, j\left(x_{n}-x\right)\right\rangle+\left(1-\beta_{n}\right)\left\langle\left(y_{n}-x\right), j\left(x_{n}-x\right)\right\rangle \\
& \leq \beta_{n}\left\|f\left(x_{n}\right)-f(x)\right\|\left\|x_{n}-x\right\|+\beta_{n}\left\langle f(x)-x, j\left(x_{n}-x\right)\right\rangle+\left(1-\beta_{n}\right)\left\|y_{n}-x\right\|\left\|x_{n}-x\right\| \\
& \leq \beta_{n} \psi\left(\left\|x_{n}-x\right\|\right)\left\|x_{n}-x\right\|+\beta_{n}\|f(x)-x\|\left\|x_{n}-x\right\|+\left(1-\beta_{n}\right)\left\|x_{n}-x\right\|^{2},
\end{aligned}
$$

and hence

$$
\left\|x_{n}-x\right\|^{2} \leq \psi\left(\left\|x_{n}-x\right\|\right)\left\|x_{n}-x\right\|+\|f(x)-x\|\left\|x_{n}-x\right\| .
$$

Therefore

$$
\eta\left(\left\|x_{n}-x\right\|\right):=\left\|x_{n}-x\right\|-\psi\left(\left\|x_{n}-x\right\|\right) \leq\|f(x)-x\|,
$$


equivalent to

$$
\left\|x_{n}-x\right\| \leq \eta^{-1}(\|f(x)-x\|) .
$$

Thus $\left\{x_{n}\right\}$ is bounded, and so are $\left\{T\left(t_{n}\right) x_{n}\right\},\left\{f\left(x_{n}\right)\right\}$, and $\left\{y_{n}\right\}$. Next, we claim that $\left\{x_{n}\right\}$ is relatively sequentially compact. Indeed, By reflexivity of $E$ and boundedness of the sequence $\left\{x_{n}\right\}$ there exists a weakly convergent subsequence $\left\{x_{n_{j}}\right\} \subset\left\{x_{n}\right\}$ such that $x_{n_{j}} \rightarrow p$ for some $p \in C$. Now we show that $p \in \operatorname{Fix}(\mathcal{S})$. Put $x_{j}=x_{n_{j}}, \gamma_{j}=\gamma_{n_{j}}, \alpha_{j}=\alpha_{n_{j}}, \beta_{j}=\beta_{n_{j}}$ and $t_{j}=t_{n_{j}}$ for $j \in \mathbb{N}$, fixed $t>0$. Notice that

$$
\begin{aligned}
\left\|x_{j}-T(t) p\right\| \leq & \sum_{k=0}^{\left[t / t_{j}\right]-1}\left\|T\left((k+1) t_{j}\right) x_{j}-T\left(k t_{j}\right) x_{j}\right\| \\
& +\left\|T\left(\left[t / t_{j}\right] t_{j}\right) x_{j}-T\left(\left[t / t_{j}\right] t_{j}\right) p\right\|+\left\|T\left(\left[t / t_{j}\right] t_{j}\right) p-T(t) p\right\| \\
\leq & {\left[t / t_{j}\right]\left\|T\left(t_{j}\right) x_{j}-x_{j}\right\|+\left\|x_{j}-p\right\| T\left(t-\left[t / t_{j}\right] t_{j}\right) p-p \| } \\
& =\left[t / t_{j}\right] \frac{\beta_{j}}{1-\alpha_{j}}\left\|x_{j}-f\left(x_{j}\right)\right\|+\left\|x_{j}-p\right\|+\left\|T\left(t-\left[t / t_{j}\right] t_{j}\right) p-p\right\| \\
& \leq \frac{t}{1-\alpha_{j}} \frac{\beta_{j}}{t_{j}}\left\|x_{j}-f\left(x_{j}\right)\right\|+\left\|x_{j}-p\right\|+\max \left\{\|T(s) p-p\|: 0 \leq s \leq t_{j}\right\} .
\end{aligned}
$$

For all $j \in \mathbb{N}$, we have

$$
\limsup _{j \rightarrow \infty}\left\|x_{j}-T(t) p\right\| \leq \limsup _{j \rightarrow \infty}\left\|x_{j}-p\right\| .
$$

Since Banach space $E$ with a weakly sequentially continuous duality mapping satisfies Opial's condition, $T(t) p=p$. Therefore $p \in \operatorname{Fix}(\mathcal{S})$. In Equation (2.4), replace $p$ with $x$ to obtain

$$
\left\|x_{j}-p\right\|\left(\left\|x_{j}-p\right\|-\psi\left(\left\|x_{j}-p\right\|\right)\right) \leq\left\langle f(p)-p, j\left(x_{j}-p\right)\right\rangle .
$$

Using that the duality map $j$ is single-valued and weakly sequentially continuous from $E$ to $E^{*}$, we get that

$$
\lim _{j \rightarrow \infty}\left\|x_{j}-p\right\|\left(\left\|x_{j}-p\right\|-\psi\left(\left\|x_{j}-p\right\|\right)\right) \leq \lim _{j \rightarrow \infty}\left\langle f(p)-p, j\left(x_{j}-p\right)\right\rangle=0 .
$$

If $\lim _{j \rightarrow \infty}\left\|x_{j}-p\right\|=0$, then we have done.

If $\lim _{j \rightarrow \infty}\left(\left\|x_{j}-p\right\|-\psi\left(\| x_{j}-p||\right)\right)=0$, then we have $\lim _{j \rightarrow \infty}\left\|x_{j}-p\right\|=\lim _{j \rightarrow \infty} \psi\left(\| x_{j}\right.$ $-p||)$.

Since $\psi$ is a continuous function, $\lim _{j \rightarrow \infty}\left\|x_{j}-p\right\|=\psi\left(\lim _{j \rightarrow \infty} \| x_{j}-p||\right)$. By Definition of $\psi$, we have $\lim _{j \rightarrow \infty}\left\|x_{j}-p\right\|=0$. Hence $\left\{x_{n}\right\}$ is relatively sequentially compact, i.e., there exists a subsequence $\left\{x_{n_{j}}\right\} \subseteq\left\{x_{n}\right\}$ such that $x_{n_{j}} \rightarrow p$ as $j \rightarrow \infty$. Next, we show that $p$ is a solution in $\operatorname{Fix}(\mathcal{S})$ to the variational inequality (2.2). In fact, for any $x \in \operatorname{Fix}(\mathcal{S})$,

$$
\begin{aligned}
\left\|x_{n}-x\right\|^{2} & =\left\langle\beta_{n} f\left(x_{n}\right)+\left(1-\beta_{n}\right) y_{n}-x, j\left(x_{n}-x\right)\right\rangle \\
& =\left\langle\beta_{n}\left(f\left(x_{n}\right)-x_{n}+x_{n}-x\right)+\left(1-\beta_{n}\right)\left(y_{n}-x\right), j\left(x_{n}-x\right)\right\rangle \\
& =\beta_{n}\left\langle f\left(x_{n}\right)-x_{n}, j\left(x_{n}-x\right)\right\rangle+\beta_{n}\left\langle x_{n}-x, j\left(x_{n}-x\right)\right\rangle+\left(1-\beta_{n}\right)\left\langle y_{n}-x, j\left(x_{n}-x\right)\right\rangle \\
& \leq \beta_{n}\left\langle f\left(x_{n}\right)-x_{n}, j\left(x_{n}-x\right)\right\rangle+\beta_{n}\left\|x_{n}-x\right\|^{2}+\left(1-\beta_{n}\right)\left\|y_{n}-x\right\|\left\|x_{n}-x\right\| \\
& \leq \beta_{n}\left\langle f\left(x_{n}\right)-x_{n}, j\left(x_{n}-x\right)\right\rangle+\beta_{n}\left\|x_{n}-x\right\|^{2}+\left(1-\beta_{n}\right)\left\|x_{n}-x\right\|^{2} .
\end{aligned}
$$

Therefore,

$$
\left\langle f\left(x_{n}\right)-x_{n}, j\left(x-x_{n}\right)\right\rangle \leq 0 .
$$


Since the sets $\left\{x_{n}-x\right\}$ and $\left\{x_{n}-f\left(x_{n}\right)\right\}$ are bounded and the duality mapping $j$ is singled-valued and weakly sequentially continuous from $E$ into $E^{*}$, for any fixed $x \in \operatorname{Fix}(\mathcal{S})$. It follows from $(2.5)$ that

$$
\langle f(p)-p, j(x-p)\rangle-\lim _{j \rightarrow \infty}\left\langle f\left(x_{n_{j}}\right)-x_{n_{j}} j\left(x-x_{n_{j}}\right)\right\rangle \leq 0, \quad \forall x \in \operatorname{Fix}(\mathcal{S}) .
$$

This is, $p \in \operatorname{Fix}(\mathcal{S})$ is a solution of the variational inequality (2.2).

Finally, we show that $p \in \operatorname{Fix}(\mathcal{S})$ is the unique solution of the variational inequality (2.2). In fact, supposing $p, q \in \operatorname{Fix}(\mathcal{S})$ satisfy the inequality (2.2) with $p \neq q$, we get that there exists $\varepsilon>0$ such that $\|p-q\| \geq \varepsilon$. By Proposition 1.4 there exists $r \in(0,1)$ such that $\|f(p)-f(q)\| \leq r\|p-q\|$. We get that

$$
\langle(f-I) p, j(q-p)\rangle \leq 0 \text { and }\langle(f-I) q, j(p-q)\rangle \leq 0 .
$$

Adding the two above inequalities, we have that

$$
0<(1-r) \varepsilon^{2} \leq(1-r)\|p-q\|^{2} \leq\langle((I-f) p-(I-f) q, j(p-q))\rangle \leq 0,
$$

which is contradiction. We must have $p=q$, and the uniqueness is proved.

In a similar way, it can be shown that each cluster point of sequence $\left\{x_{n}\right\}$ is equal to $q$. Therefore, the entire sequence $\left\{x_{n}\right\}$ converges to $q$ and the proof is complete.

Setting $f$ is a contraction on $C$ in Theorem 2.1, we have the following results immediately.

Corollary 2.2. [11, Theorem 3.1] Let E be a reflexive Banach space which admits a weakly sequenctially continuous duality mapping $J$ from $E$ into $E^{*}$, suppose $C$ is a nonempty closed convex subset of $E$. Let $\mathcal{S}:=\{T(t): t \geq 0\}$ be a nonexpansive semigroup on $C$ such that $\operatorname{Fix}(\mathcal{S}) \neq \emptyset$, and $f: C \rightarrow C$ a contraction on $C$. Let $\left\{\alpha_{n}\right\} \subset(0,1),\left\{\beta_{n}\right\} \subset$ $(0,1)$, and $\left\{t_{n}\right\} \subset(0, \infty)$ be sequences of real numbers satisfying $\lim _{n \rightarrow \infty} \alpha_{n}=\lim _{n \rightarrow \infty} t_{n}=\lim _{n \rightarrow \infty} \frac{\beta_{n}}{t_{n}}=0$. Define a sequence $\left\{x_{n}\right\}$ in $C$ by

$$
\left\{\begin{array}{l}
y_{n}=\alpha_{n} x_{n}+\left(1-\alpha_{n}\right) T\left(t_{n}\right) x_{n} \\
x_{n}=\beta_{n} f\left(x_{n}\right)+\left(1-\beta_{n}\right) y_{n}, \quad \text { for all } n \geq 1
\end{array}\right.
$$

Then $\left\{x_{n}\right\}$ converges strongly to $q$, as $n \rightarrow \infty q$ is the element of $\operatorname{Fix}(\mathcal{S})$ such that $q$ is the unique solution in $\operatorname{Fix}(\mathcal{S})$ to the following variational inequality:

$$
\langle(f-I) q, j(x-q)\rangle \leq 0 \quad \text { for all } x \in \operatorname{Fix}(\mathcal{S}) .
$$

Theorem 2.3. Let E be a reflexive Banach space which admits a weakly sequenctially continuous duality mapping $J$ from $E$ into $E^{*}$, suppose $C$ is a nonempty closed convex subset of $E$. Let $\{T(t): t \geq 0\}$, be a nonexpansive semigroup on $C$ such that $\operatorname{Fix}(\mathcal{S}) \neq \emptyset$, and $f: C \rightarrow C$ be a generalized contraction on $C$. Let $\left\{\alpha_{n}\right\} \subset(0,1),\left\{\beta_{n}\right\} \subset(0,1),\left\{\gamma_{n}\right\} \subset$ $[0,1]$, and $\left\{t_{n}\right\} \subset(0, \infty)$ be sequences of real numbers satisfying the conditions:

$$
\begin{aligned}
& \text { (C1) } \lim _{n \rightarrow \infty} \beta_{n}=0, \sum_{n=0}^{\infty} \beta_{n}=\infty \text { and } \lim _{n \rightarrow \infty} t_{n}=0, \\
& \text { (C2) } \lim _{n \rightarrow \infty} \alpha_{n}=0 \text { and } \lim _{n \rightarrow \infty} \gamma_{n}=1, \\
& \text { (C3) } \sum_{n=0}^{\infty}\left|\alpha_{n+1}-\alpha_{n}\right|<\infty, \sum_{n=0}^{\infty}\left|\beta_{n+1}-\beta_{n}\right|<\infty \text {, and } \sum_{n=0}^{\infty}\left|\gamma_{n+1}-\gamma_{n}\right|<\infty .
\end{aligned}
$$




$$
\left\{\begin{array}{l}
x_{0} \in C^{\prime} \\
z_{n}=\gamma_{n} x_{n}+\left(1-\gamma_{n}\right) T\left(t_{n}\right) x_{n} \\
y_{n}=\alpha_{n} x_{n}+\left(1-\alpha_{n}\right) T\left(t_{n}\right) z_{n} \\
x_{n+1}=\beta_{n} f\left(x_{n}\right)+\left(1-\beta_{n}\right) y_{n}, \quad n \geq 0 .
\end{array}\right.
$$

Suppose

$$
\sum_{n=0}^{\infty} \sup _{x \in \tilde{C}}\left\|T\left(t_{n}\right) x-T\left(t_{n-1}\right) x\right\|<\infty,
$$

where $\tilde{C}$ is any bounded subset of $C$. Then $\left\{x_{n}\right\}$ converges strongly to $q$, as $n \rightarrow \infty$ where $q$ is the unique solution in $\operatorname{Fix}(\mathcal{S})$ to the variational inequality (2.2).

Proof. First, we show that $\left\{x_{n}\right\}$ is bounded. Indeed, if we take a fixed point $x \in \operatorname{Fix}(\mathcal{S})$. We will prove by induction that

$$
\left\|x_{n}-x\right\| \leq M \text { for all } n \geq 0,
$$

where $M:=\left\{|| x_{0}-z||, \eta^{-1}(|| f(x)-x||)\right\}$. From Definition of (2.8), notice that

$$
\left\|z_{n}-x\right\| \leq \gamma_{n}\left\|x_{n}-x\right\|+\left(1-\gamma_{n}\right)\left\|T\left(t_{n}\right) x_{n}-x\right\| \leq\left\|x_{n}-x\right\| .
$$

It follows that

$$
\left\|y_{n}-x\right\| \leq \alpha_{n}\left\|x_{n}-x\right\|+\left(1-\alpha_{n}\right)\left\|T\left(t_{n}\right) z_{n}-x\right\| \leq \alpha_{n}\left\|x_{n}-x\right\|+\left(1-\alpha_{n}\right)\left\|x_{n}-x\right\| \leq\left\|x_{n}-x\right\| .
$$

The case $n=0$ is obvious.

Suppose that $\left\|x_{n}-x\right\| \leq M$, we have

$$
\begin{aligned}
\left\|x_{n+1}-x\right\| & \leq \beta_{n}\left\|f\left(x_{n}\right)-x\right\|+\left(1-\beta_{n}\right)\left\|y_{n}-x\right\| \\
& \leq \beta_{n}\left\|f\left(x_{n}\right)-f(x)\right\|+\beta_{n}\|f(x)-x\|+\left(1-\beta_{n}\right)\left\|y_{n}-x\right\| \\
& \leq \beta_{n} \psi\left(\left\|x_{n}-x\right\|\right)+\beta_{n}\|f(x)-x\|+\left(1-\beta_{n}\right)\left\|x_{n}-x\right\| \\
& =\beta_{n} \psi\left(\left\|x_{n}-x\right\|\right)+\beta_{n} \eta\left(\eta^{-1}(\|f(x)-x\|)\right)+\left(1-\beta_{n}\right)\left\|x_{n}-x\right\| \\
& \leq \beta_{n} \psi(M)+\beta_{n} \eta(M)+\left(1-\beta_{n}\right) M \\
& =\beta_{n} \psi(M)+\beta_{n}(M-\psi(M))+\left(1-\beta_{n}\right) M=M .
\end{aligned}
$$

By induction,

$$
\left\|x_{n}-x\right\| \leq \max \left\{\left\|x_{0}-x\right\|, \eta^{-1}(\|f(x)-x\|)\right\}, \quad \forall n \geq 0 .
$$

Thus $\left\{x_{n}\right\}$ is bounded, and so are $\left\{T\left(t_{n}\right) x_{n}\right\},\left\{y_{n}\right\},\left\{z_{n}\right\},\left\{T\left(t_{n}\right) z_{n}\right\}$, and $\left\{f\left(x_{n}\right)\right\}$. As a result, we obtain by condition (C1),

$$
\left\|x_{n+1}-y_{n}\right\|=\beta_{n}\left\|f\left(x_{n}\right)-y_{n}\right\| \rightarrow 0 .
$$

We next show that

$$
\left\|x_{n}-T\left(t_{n}\right) x_{n}\right\| \rightarrow 0 .
$$

It suffices to show that

$$
\left\|x_{n+1}-x_{n}\right\| \rightarrow 0 \text {. }
$$


Indeed, if (2.9) holds, then noting (2.7), we obtain

$$
\begin{aligned}
\left\|x_{n}-T\left(t_{n}\right) x_{n}\right\| & \leq\left\|x_{n}-x_{n+1}\right\|+\left\|x_{n+1}-y_{n}\right\|+\left\|y_{n}-T\left(t_{n}\right) z_{n}\right\|+\left\|T\left(t_{n}\right) z_{n}-T\left(t_{n}\right) x_{n}\right\| \\
& \leq\left\|x_{n}-x_{n+1}\right\|+\left\|x_{n+1}-y_{n}\right\|+\alpha_{n}\left\|x_{n}-T\left(t_{n}\right) z_{n}\right\|+\left\|z_{n}-x_{n}\right\| \\
& \leq\left\|x_{n}-x_{n+1}\right\|+\left\|x_{n+1}-y_{n}\right\|+\alpha_{n}\left\|x_{n}-T\left(t_{n}\right) x_{n}\right\|+\alpha_{n}\left\|T\left(t_{n}\right) x_{n}-T\left(t_{n}\right) z_{n}\right\| \\
& +\left\|z_{n}-x_{n}\right\| \\
& \leq\left\|x_{n}-x_{n+1}\right\|+\left\|x_{n+1}-y_{n}\right\|+\alpha_{n}\left\|x_{n}-T\left(t_{n}\right) x_{n}\right\|+\alpha_{n}\left\|x_{n}-z_{n}\right\|+\left\|z_{n}-x_{n}\right\| \\
& \leq\left\|x_{n}-x_{n+1}\right\|+\left\|x_{n+1}-y_{n}\right\|+\alpha_{n}\left\|x_{n}-T\left(t_{n}\right) x_{n}\right\|+\left(1+\alpha_{n}\right)\left\|x_{n}-z_{n}\right\| \\
& \leq\left\|x_{n}-x_{n+1}\right\|+\left\|x_{n+1}-y_{n}\right\|+\alpha_{n}\left\|x_{n}-T\left(t_{n}\right) x_{n}\right\| \\
& +\left(1+\alpha_{n}\right)\left(1-\gamma_{n}\right)\left\|x_{n}-T\left(t_{n}\right) x_{n}\right\| .
\end{aligned}
$$

It follows from $(\mathrm{C} 2)$ that

$$
\begin{aligned}
\left\|x_{n}-T\left(t_{n}\right) x_{n}\right\| \leq & \left\|x_{n}-x_{n+1}\right\|+\left\|x_{n+1}-y_{n}\right\|+\alpha_{n}\left\|x_{n}-T\left(t_{n}\right) x_{n}\right\| \\
& +\left(1+\alpha_{n}\right)\left(1-\gamma_{n}\right)\left\|x_{n}-T\left(t_{n}\right) x_{n}\right\| \rightarrow 0 \text { as } n \rightarrow \infty .
\end{aligned}
$$

Suppose that (2.9) is not holds, there exists $\varepsilon>0$ and subsequence $\left\|x_{n_{j}+1}-x_{n_{j}}\right\|$ of $\|$ $x_{n}+1_{1}-x_{n} \|$ such that $\left\|x_{n_{j}+1}-x_{n_{j}}\right\| \geq \varepsilon$ for all $j \in \mathbb{N}$. By Proposition 1.4, there exists $r \in$ $(0,1)$ such that $\left\|f\left(x_{n_{j}+1}\right)-f\left(x_{n_{j}}\right)\right\| \leq r\left\|x_{n_{j}+1}-x_{n_{j}}\right\|$ for all $j \in \mathbb{N}$. Put $x_{j}=x_{n_{j}}, \alpha_{j}=\alpha_{n_{j}}, \beta_{j}=\beta_{n_{j}}, \gamma_{j}=\gamma_{n_{j}}$ and $t_{j}=t_{n_{j}}$ for $j \in \mathbb{N}$. We calculate $x_{j+1}-x_{j}$. Observing that

$$
x_{j+1}=\beta_{j} f\left(x_{j}\right)+\left(1-\beta_{j}\right) y_{j} \quad \text { and } \quad x_{j}=\beta_{j-1} f\left(x_{j-1}\right)+\left(1-\beta_{j-1}\right) y_{j-1},
$$

we get

$$
\begin{aligned}
x_{j+1}-x_{j}= & \beta_{j} f\left(x_{j}\right)+\left(1-\beta_{j}\right) y_{j}-\beta_{j-1} f\left(x_{j-1}\right)-\left(1-\beta_{j-1}\right) y_{j-1} \\
= & \beta_{j}\left(f\left(x_{j}\right)-f\left(x_{j-1}\right)\right)+\left(\beta_{j}-\beta_{j-1}\right) f\left(x_{j-1}\right)+\left(1-\beta_{j}\right)\left(y_{j}-y_{j-1}\right) \\
& -\left(\beta_{j}-\beta_{j-1}\right) y_{j-1} .
\end{aligned}
$$

That is

$$
\begin{aligned}
\left\|x_{j+1}-x_{j}\right\| \leq & \beta_{j} r\left\|x_{j}-x_{j-1}\right\|+\left|\beta_{j}-\beta_{j-1}\right|\left\|f\left(x_{j-1}\right)\right\|+\left(1-\beta_{j}\right)\left\|y_{j}-y_{j-1}\right\|_{(2.1} \\
& +\left|\beta_{j}-\beta_{j-1}\right|\left\|y_{j-1}\right\| .
\end{aligned}
$$

Noticing that

$$
y_{j}=\alpha_{j} x_{j}+\left(1-\alpha_{j}\right) T\left(t_{j}\right) z_{j} \quad \text { and } \quad y_{j-1}=\alpha_{j-1} x_{j-1}+\left(1-\alpha_{j-1}\right) T\left(t_{j-1}\right) z_{j-1} .
$$

We obtain that

$$
\begin{aligned}
y_{j}-y_{j-1}= & \alpha_{j} x_{j}+\left(1-\alpha_{j}\right) T\left(t_{j}\right) z_{j}-\alpha_{j-1} x_{j-1}-\left(1-\alpha_{j-1}\right) T\left(t_{j-1}\right) z_{j-1} . \\
= & \alpha_{j}\left(x_{j}-x_{j-1}\right)+\left(\alpha_{j}-\alpha_{j-1}\right) x_{j-1} \\
& +\left(1-\alpha_{j-1}\right)\left(T\left(t_{j}\right) z_{j-1}-T\left(t_{j-1}\right) z_{j-1}\right)+\left(1-\alpha_{j}\right)\left(T\left(t_{j}\right) z_{j}-T\left(t_{j}\right) z_{j-1}\right) \\
& -\left(\alpha_{j}-\alpha_{j-1}\right) T\left(t_{j}\right) z_{j-1} .
\end{aligned}
$$

This implied that

$$
\begin{aligned}
\left\|y_{j}-y_{j-1}\right\| \leq & \alpha_{j}\left\|x_{j}-x_{j-1}\right\|+\left|\alpha_{j}-\alpha_{j-1}\right|\left\|x_{j-1}\right\| \\
& +\left(1-\alpha_{j-1}\right)\left\|T\left(t_{j}\right) z_{j-1}-T\left(t_{j-1}\right) z_{j-1}\right\|+\left(1-\alpha_{j}\right)\left\|z_{j}-z_{j-1}\right\|(2.13) \\
& +\left|\alpha_{j}-\alpha_{j-1}\right|\left\|T\left(t_{j}\right) z_{j-1}\right\| .
\end{aligned}
$$


Again from (2.6) we obtain

$$
\begin{aligned}
z_{j}-z_{j-1}= & \gamma_{j}\left(x_{j}-x_{j-1}\right)+\left(1-\gamma_{j}\right)\left(T\left(t_{j}\right) x_{j}-T\left(t_{j}\right) x_{j-1}\right) \\
& +\left(1-\gamma_{j}\right)\left(T\left(t_{j}\right) x_{j-1}-T\left(t_{j-1}\right) x_{j-1}\right)+\left(\gamma_{j}-\gamma_{j-1}\right)\left(x_{j-1}-T\left(t_{j-1}\right) x_{j-1}\right),
\end{aligned}
$$

that is,

$$
\begin{aligned}
\left\|z_{j}-z_{j-1}\right\| \leq & \gamma_{j}\left\|x_{j}-x_{j-1}\right\|+\left(1-\gamma_{j}\right)\left\|T\left(t_{j}\right) x_{j}-T\left(t_{j}\right) x_{j-1}\right\| \\
& +\left(1-\gamma_{j}\right)\left\|T\left(t_{j}\right) x_{j-1}-T\left(t_{j-1}\right) x_{j-1}\right\|+\left|\gamma_{j}-\gamma_{j-1}\right|\left\|x_{j-1}-T\left(t_{j-1}\right) x_{j-1}\right\| \\
\leq & \left\|x_{j}-x_{j-1}\right\|+\left(1-\gamma_{j}\right)\left\|T\left(t_{j}\right) x_{j-1}-T\left(t_{j-1}\right) x_{j-1}\right\| \\
& +\left|\gamma_{j}-\gamma_{j-1}\right|\left\|x_{j-1}-T\left(t_{j-1}\right) x_{j-1}\right\| .
\end{aligned}
$$

Substituting (2.14) into (2.13),

$$
\begin{aligned}
\left\|y_{j}-y_{j-1}\right\| \leq & \alpha_{j}\left\|x_{j}-x_{j-1}\right\|+\left|\alpha_{j}-\alpha_{j-1}\right|\left\|x_{j-1}\right\| \\
& +\left(1-\alpha_{j-1}\right)\left\|T\left(t_{j}\right) z_{j-1}-T\left(t_{j-1}\right) z_{j-1}\right\|+\left|\alpha_{j}-\alpha_{j-1}\right|\left\|T\left(t_{j}\right) z_{j-1}\right\| \\
& +\left(1-\alpha_{j}\right)\left\|x_{j}-x_{j-1}\right\|+\left(1-\alpha_{j}\right)\left(1-\gamma_{j}\right)\left\|T\left(t_{j}\right) x_{j-1}-T\left(t_{j-1}\right) x_{j-1}\right\| \\
& \left.+\left(1-\alpha_{j}\right)\left|\gamma_{j}-\gamma_{j-1}\right|\left\|x_{j-1}-T\left(t_{j-1}\right) x_{j-1}\right\|\right] \\
\leq & \left\|x_{j}-x_{j-1}\right\|+\left|\alpha_{j}-\alpha_{j-1}\right|\left\|x_{j-1}\right\| \\
& +\left\|T\left(t_{j}\right) z_{j-1}-T\left(t_{j-1}\right) z_{j-1}\right\|+\left|\alpha_{j}-\alpha_{j-1}\right|\left\|T\left(t_{j}\right) z_{j-1}\right\| \\
& +\left\|T\left(t_{j}\right) x_{j-1}-T\left(t_{j-1}\right) x_{j-1}\right\| \\
& +\left|\gamma_{j}-\gamma_{j-1}\right|\left\|x_{j-1}-T\left(t_{j-1}\right) x_{j-1}\right\| .
\end{aligned}
$$

Substituting (2.15) into (2.11),

$$
\begin{aligned}
\left\|x_{j+1}-x_{j}\right\| \leq & \beta_{j} r\left\|x_{j}-x_{j-1}\right\|+\left|\beta_{j}-\beta_{j-1}\right|\left\|f\left(x_{j-1}\right)\right\|+\left(1-\beta_{j}\right)\left\|x_{j}-x_{j-1}\right\| \\
& +\left(1-\beta_{j}\right)\left|\alpha_{j}-\alpha_{j-1}\right|\left\|x_{j-1}\right\| \\
& +\left(1-\beta_{j}\right)\left\|T\left(t_{j}\right) z_{j-1}-T\left(t_{j-1}\right) z_{j-1}\right\|+\left(1-\beta_{j}\right) \mid \alpha_{j}-\alpha_{j-1}\left\|T\left(t_{j}\right) z_{j-1}\right\| \\
& +\left(1-\beta_{j}\right)\left\|T\left(t_{j}\right) x_{j-1}-T\left(t_{j-1}\right) x_{j-1}\right\| \\
& +\left(1-\beta_{j}\right)\left|\gamma_{j}-\gamma_{j-1}\right|\left\|x_{j-1}-T\left(t_{j-1}\right) x_{j-1}\right\| \\
& +\left|\beta_{j}-\beta_{j-1}\right|\left\|\gamma_{j-1}\right\| \\
\leq & \left(1-(1-r) \beta_{j}\right)\left\|x_{j}-x_{j-1}\right\|+\left|\beta_{j}-\beta_{j-1}\right|\left\|f\left(x_{j-1}\right)\right\| \\
& +\left|\alpha_{j}-\alpha_{j-1}\right|\left\|x_{j-1}\right\| \\
& +\left\|T\left(t_{j}\right) z_{j-1}-T\left(t_{j-1}\right) z_{j-1}\right\|+\left|\alpha_{j}-\alpha_{j-1}\right|\left\|T\left(t_{j}\right) z_{j-1}\right\| \\
& +\left\|T\left(t_{j}\right) x_{j-1}-T\left(t_{j-1}\right) x_{j-1}\right\| \\
& +\left|\gamma_{j}-\gamma_{j-1}\right|\left\|x_{j-1}-T\left(t_{j-1}\right) x_{j-1}\right\| \\
& +\left|\beta_{j}-\beta_{j-1}\right|\left\|y_{j-1}\right\| .
\end{aligned}
$$

Hence,

$$
\begin{aligned}
\left\|x_{j+1}-x_{j}\right\| \leq & \left(1-(1-r) \beta_{j}\right)\left\|x_{j}-x_{j-1}\right\|+\left(2\left|\alpha_{j}-\alpha_{j-1}\right|+2\left|\beta_{j}-\beta_{j-1}\right|+\left|\gamma_{j}-\gamma_{j-1}\right|\right) M \\
& +\sup _{x \in\left\{x_{n}\right\}}\left\|T\left(t_{j}\right) x-T\left(t_{j-1}\right) x\right\|+\sup _{z \in\left\{z_{n}\right\}}\left\|T\left(t_{j}\right) z-T\left(t_{j-1}\right) z\right\|,
\end{aligned}
$$

where $M \geq \max \left\{|| x_{j-1}-T\left(t_{j-1}\right) x_{j-1}||,|| y_{j-1}||,|| x_{j-1}||,|| T\left(t_{j}\right) z_{j-1}||,|| f\left(x_{j-1}\right)||\right\}$ for all $j$.

By assumption, we have that $\sum_{j=1}^{\infty} \beta_{j}=\infty$, and $\sum_{j=1}^{\infty}\left(2\left|\alpha_{j}-\alpha_{j-1}\right|+2\left|\beta_{j}-\beta_{j-1}\right|+\left|\gamma_{j}-\gamma_{j-1}\right|+\sup _{x \in\left\{x_{n}\right\}}\left\|T\left(t_{j}\right) x-T\left(t_{j-1}\right) x\right\|+\sup _{z \in\left\{z_{n}\right]}\left\|T\left(t_{j}\right) z-T\left(t_{j-1}\right) z\right\|\right)<\infty$. Hence, Lemma 1.7 is applicable to (2.17) and we obtain ||$x_{j+1}-x_{j}|| \rightarrow 0$, which is a contradiction. So (2.9) is proved. Applying Theorem 2.1, there is a unique solution $q \in \operatorname{Fix}(\mathcal{S})$ to the following variational inequality:

$$
\langle f(q)-q, j(x-q)\rangle \leq 0 \quad \text { for all } x \in \operatorname{Fix}(\mathcal{S}) .
$$


Next, we show that

$$
\limsup _{n \rightarrow \infty}\left\langle f(q)-q, j\left(x_{n+1}-q\right)\right\rangle \leq 0 .
$$

Indeed, we can take a subsequence $\left\{x_{n_{i}}\right\}$ of $\left\{x_{n}\right\}$ such that

$$
\limsup _{n \rightarrow \infty}\left\langle f(q)-q, j\left(x_{n+1}-q\right)\right\rangle=\lim _{i \rightarrow \infty}\left\langle f(q)-q, j\left(x_{n_{i}+1}-q\right)\right\rangle .
$$

By the reflexivity of $E$ and boundedness of the sequence $\left\{x_{n}\right\}$, we may assume, without loss of generality, that $x_{n_{i}} \rightarrow p$ for some $p \in C$. Now we show that $p \in \operatorname{Fix}(\mathcal{S})$. Put $x_{i}=x_{n_{i}}, \alpha_{i}=\alpha_{n_{i}}, \beta_{i}=\beta_{n_{i}}$ and $t_{i}=t_{n_{i}}$ for $i \in \mathbb{N}$, let $t_{i} \geq 0$ be such that

$$
t_{i} \rightarrow 0 \quad \text { and } \quad \frac{\left\|T\left(t_{i}\right) x_{i}-x_{i}\right\|}{t_{i}} \rightarrow 0, \quad i \rightarrow \infty .
$$

Fix $t>0$. Notice that

$$
\begin{aligned}
\left\|x_{i}-T(t) p\right\| \leq & \sum_{k=0}^{\left[t / t_{i}\right]-1}\left\|T\left((k+1) t_{i}\right) x_{i}-T\left(k t_{i}\right) x_{i}\right\| \\
& +\left\|T\left(\left[t / t_{i}\right] t_{i}\right) x_{i}-T\left(\left[t / t_{i}\right] t_{i}\right) p\right\|+\left\|T\left(\left[t / t_{i}\right] t_{i}\right) p-T(t) p\right\| \\
\leq & {\left[t / t_{i}\right]\left\|T\left(t_{i}\right) x_{i}-x_{i}\right\|+\left\|x_{i}-p\right\|+\left\|T\left(t-\left[t / t_{i}\right] t_{i}\right) p-p\right\| } \\
\leq & t \frac{\left\|T\left(t_{i}\right) x_{i}-x_{i}\right\|}{t_{i}}+\left\|x_{i}-p\right\|+\left\|T\left(t-\left[t / t_{i}\right] t_{i}\right) p-p\right\| \\
\leq & t \frac{\left\|T\left(t_{i}\right) x_{i}-x_{i}\right\|}{t_{i}}+\left\|x_{i}-p\right\|+\max \left\{\|T(s) p-p\|: 0 \leq s \leq t_{i}\right\} .
\end{aligned}
$$

For all $i \in \mathbb{N}$, we have

$$
\limsup _{i \rightarrow \infty}\left\|x_{i}-T(t) p\right\| \leq \limsup _{i \rightarrow \infty}\left\|x_{i}-p\right\| .
$$

Since Banach space $E$ with a weakly sequentially continuous duality mapping satisfies Opial's condition, this implies $T(t) p=p$. Therefore $p \in \operatorname{Fix}(\mathcal{S})$. In view of the variational inequality (2.2) and the assumption that duality mapping $J$ is weakly sequentially continuous, we conclude

$$
\begin{aligned}
\limsup _{n \rightarrow \infty}\left\langle f(q)-q_{1} j\left(x_{n+1}-q\right)\right\rangle & =\lim _{i \rightarrow \infty}\left\langle f(q)-q, j\left(x_{n_{i}+1}-q\right)\right\rangle \\
& =\langle f(q)-q, j(p-q)\rangle \leq 0 .
\end{aligned}
$$

Then (2.18) is proved. Finally show that $x_{n} \rightarrow q$, i.e. $\left\|x_{n}-q\right\| \rightarrow 0$. Suppose that $\| x_{n}$ - $q \| \rightarrow 0$, then there exists $\varepsilon>0$ and a subsequence $\left\{x_{n_{j}}\right\}$ of $\left\{x_{n}\right\}$ such that $\left\|x_{n_{j}}-q\right\| \geq \varepsilon$ for all $j \in \mathbb{N}$. Put $x_{j}=x_{n_{j}}, \alpha_{j}=\alpha_{n_{j}}, \beta_{j}=\beta_{n_{j}}$ and $t_{j}=t_{n_{j}}$ for $j \in \mathbb{N}$. By Proposition 1.4, there exists $r \in(0,1)$ such that $\left\|f\left(x_{j}\right)-f(q)\right\| \leq r\left\|x_{j}-q\right\|$ for all $j \in \mathbb{N}$. As a matter of fact, from Lemma 1.6 we have that 


$$
\begin{aligned}
\left\|x_{j+1}-q\right\|^{2}= & \left\|\beta_{j} f\left(x_{j}\right)+\left(1-\beta_{j}\right)\left(\alpha_{j} x_{j}+\left(1-\alpha_{j}\right) T\left(t_{j}\right) z_{j}\right)-q\right\|^{2} \\
= & \|\left(1-\beta_{j}\right)\left(\alpha_{j}\left(x_{j}-q\right)+\left(1-\alpha_{j}\left(T\left(t_{j}\right) z_{j}-q\right)\right)+\beta_{j}\left(f\left(x_{j}\right)-q\right) \|^{2}\right. \\
\leq & \left(1-\beta_{j}\right)^{2}\left\|\alpha_{j}\left(x_{j}-q\right)+\left(1-\alpha_{j}\right)\left(T\left(t_{j}\right) z_{j}-q\right)\right\|^{2}+2 \beta_{j}\left\langle f\left(x_{j}\right)-q, j\left(x_{j+1}-q\right)\right\rangle \\
\leq & \left(1-\beta_{j}\right)^{2}\left(\alpha_{j}\left\|x_{j}-q\right\|+\left(1-\alpha_{j}\right)\left\|T\left(t_{j}\right) z_{j}-q\right\|\right)^{2} \\
& +2 \beta_{j}\left\langle f\left(x_{j}\right)-f(q), j\left(x_{j+1}-q\right)\right\rangle+2 \beta_{j}\left\langle f(q)-q, j\left(x_{j+1}-q\right)\right\rangle \\
\leq & \left(1-\beta_{j}\right)^{2}\left\|x_{j}-q\right\|^{2}+2 \beta_{j}\left\|f\left(x_{j}\right)-f(q)\right\|\left\|x_{j+1}-q\right\|+2 \beta_{j}\left\langle f(q)-q, j\left(x_{j+1}-q\right)\right\rangle \\
\leq & \left(1-\beta_{j}\right)^{2}\left\|x_{j}-q\right\|^{2}+2 \beta_{j} r\left\|x_{j}-q\right\|\left\|x_{j+1}-q\right\|+2 \beta_{j}\left\langle f(q)-q, j\left(x_{j+1}-q\right)\right\rangle \\
\leq & \left(1-\beta_{j}\right)^{2}\left\|x_{j}-q\right\|^{2}+\beta_{j} r\left(\left\|x_{j}-q\right\|^{2}+\left\|x_{j+1}-q\right\|^{2}\right)+2 \beta_{j}\left\langle f(q)-q, j\left(x_{j+1}-q\right)\right\rangle \\
\leq & \left(\left(1-\beta_{j}\right)^{2}+\beta_{j} r\right)\left\|x_{j}-q\right\|^{2}+\beta_{j} r\left\|x_{j+1}-q\right\|^{2}+2 \beta_{j}\left\langle f(q)-q, j\left(x_{j+1}-q\right)\right\rangle .
\end{aligned}
$$

It follows that

$$
\begin{aligned}
\left\|x_{j+1}-q\right\|^{2} & \leq \frac{\left(1-(2-r) \beta_{j}+\beta_{j}^{2}\right)}{1-\beta_{j} r}\left\|x_{j}-q\right\|^{2}+\frac{2 \beta_{j}}{1-\beta_{j} r}\left\langle f(q)-q, j\left(x_{j+1}-q\right)\right\rangle \\
& =\frac{1-(2-r) \beta_{j}}{1-\beta_{j} r}\left\|x_{j}-q\right\|^{2}+\frac{\beta_{j}^{2}}{1-\beta_{j} r}\left\|x_{j}-q\right\|^{2}+\frac{2 \beta_{j}}{1-\beta_{j} r}\left\langle f(q)-q, j\left(x_{j+1}-q\right)\right\rangle \\
& \leq \frac{1-(2-r) \beta_{j}}{1-\beta_{j} r}\left\|x_{j}-q\right\|^{2}+\frac{\beta_{j}^{2}}{1-r}\left\|x_{j}-q\right\|^{2}+\frac{2 \beta_{j}}{1-r}\left\langle f(q)-q, j\left(x_{j+1}-q\right)\right\rangle \\
& \leq \frac{1-\beta_{j} r-2(1-r) \beta_{j}}{1-\beta_{j} r}\left\|x_{j}-q\right\|^{2}+\beta_{j}^{2} M+\frac{2 \beta_{j}}{1-r}\left\langle f(q)-q, j\left(x_{j+1}-q\right)\right\rangle \\
& =\left(1-\frac{2(1-r) \beta_{j}}{1-\beta_{j} r}\right)\left\|x_{j}-q\right\|^{2}+\beta_{j}^{2} M+\frac{2 \beta_{j}}{1-r}\left\langle f(q)-q, j\left(x_{j+1}-q\right)\right\rangle \\
& \leq\left(1-2(1-r) \beta_{j}\right)\left\|x_{j}-q\right\|^{2}+\beta_{j}\left(\frac{2}{1-r}\left\langle f(q)-q, j\left(x_{j+1}-q\right)\right\rangle+\beta_{j} M\right),
\end{aligned}
$$

where $M>0$ such that $M \geq \frac{1}{1-r}\left\|x_{j}-q\right\|^{2}$. That is,

$$
\left\|x_{j+1}-q\right\|^{2} \leq\left(1-\gamma_{j}\right)\left\|x_{j}-q\right\|^{2}+\delta_{j}
$$

where $\gamma_{j}=2(1-r) \beta_{j}$ and $\frac{\delta_{j}}{\gamma_{j}}=\frac{1}{(1-r)^{2}}\left\langle f(q)-q_{,} j\left(x_{j+1}-q\right)\right\rangle+\frac{M}{2(1-r)} \beta_{j}$.

It follows by condition (B1) that $\gamma_{j} \rightarrow 0$ and $\sum_{j=1}^{\infty} \gamma_{j}=\infty$. From (2.18) we have

$$
\begin{aligned}
\limsup _{j \rightarrow \infty} \frac{\delta_{j}}{\gamma_{j}} & \leq \limsup _{j \rightarrow \infty} \frac{1}{(1-r)^{2}}\left\langle f(q)-q_{,} j\left(x_{j+1}-q\right)\right\rangle+\lim _{j \rightarrow \infty} \frac{M}{2(1-r)} \beta_{j} \\
& \leq \limsup _{j \rightarrow \infty} \frac{1}{(1-r)^{2}}\left\langle f(q)-q_{,} j\left(x_{j+1}-q\right)\right\rangle \leq 0 .
\end{aligned}
$$

Using Lemma 1.7 onto (2.19), we conclude that $\left\|x_{j}-q\right\| \rightarrow 0$. This is a contradiction. Hence $x_{n} \rightarrow q$.

The proof is completed.

If $\gamma_{n} \equiv 1$, then we have the following Corollary.

Corollary 2.4. Let E be a reflexive Banach space which admits a weakly sequenctially continuous duality mapping J from $E$ into $E^{*}$, suppose $C$ is a nonempty closed convex subset of E. Let $\{T(t): t \geq 0\}$ be a nonexpansive semigroup on $C$ such that $\operatorname{Fix}(\mathcal{S}) \neq \emptyset$, and $f: C \rightarrow C$ be a generalized contraction on $C$. Let $\left\{\alpha_{n}\right\} \subset(0,1),\left\{\beta_{n}\right\} \subset(0,1)$, and $\left\{t_{n}\right\}$ $\subset(0, \infty)$ be sequences of real numbers satisfying the conditions:

(C1) $\lim _{n \rightarrow \infty} \beta_{n}=0, \sum_{n=0}^{\infty} \beta_{n}=\infty$ and $\lim _{n \rightarrow \infty} t_{n}=0$,

(C2) $\lim _{n \rightarrow \infty} \alpha_{n}=0$,

(C3) $\sum_{n=0}^{\infty}\left|\alpha_{n+1}-\alpha_{n}\right|<\infty, \sum_{n=0}^{\infty}\left|\beta_{n+1}-\beta_{n}\right|<\infty$. 
Define a sequence $\left\{x_{n}\right\}$ in $C$ by

$$
\left\{\begin{array}{l}
x_{0} \in C \\
y_{n}=\alpha_{n} x_{n}+\left(1-\alpha_{n}\right) T\left(t_{n}\right) x_{n}, \\
x_{n+1}=\beta_{n} f\left(x_{n}\right)+\left(1-\beta_{n}\right) y_{n,} \quad n \geq 0 .
\end{array}\right.
$$

Suppose $\sum_{n=0}^{\infty}\left\|T\left(t_{n}\right) x_{n-1}-T\left(t_{n-1}\right) x_{n-1}\right\|<\infty$. Then $\left\{x_{n}\right\}$ converges strongly to $q$, as $n \rightarrow \infty$ where $q$ is the unique solution in $\operatorname{Fix}(\mathcal{S})$ to the variational inequality (2.2).

Setting $f$ is a contraction on $C$ in Corollary 2.4, we have the following results immediately.

Corollary 2.5. [11, Theorem 3.2] Let E be a reflexive Banach space which admits a weakly sequenctially continuous duality mapping $J$ from $E$ into $E^{*}$, suppose $C$ is a nonempty closed convex subset of $E$. Let $\{T(t): t \geq 0\}$ be a nonexpansive semigroup on $C$ such that $\operatorname{Fix}(\mathcal{S}) \neq \emptyset$, and $f: C \rightarrow C$ be a contraction on $C$. Let $\left\{\alpha_{n}\right\} \subset(0,1),\left\{\beta_{n}\right\} \subset$ $(0,1)$, and $\left\{t_{n}\right\} \subset(0, \infty)$ be sequences of real numbers satisfying the conditions:

(C1) $\lim _{n \rightarrow \infty} \beta_{n}=0, \sum_{n=0}^{\infty} \beta_{n}=\infty$ and $\lim _{n \rightarrow \infty} t_{n}=0$,

(C2) $\lim _{n \rightarrow \infty} \alpha_{n}=0$,

(C3) $\sum_{n=0}^{\infty}\left|\alpha_{n+1}-\alpha_{n}\right|<\infty, \sum_{n=0}^{\infty}\left|\beta_{n+1}-\beta_{n}\right|<\infty$.

Define a sequence $\left\{x_{n}\right\}$ in $C$ by

$$
\left\{\begin{array}{l}
x_{0} \in C \\
y_{n}=\alpha_{n} x_{n}+\left(1-\alpha_{n}\right) T\left(t_{n}\right) x_{n}, \\
x_{n+1}=\beta_{n} f\left(x_{n}\right)+\left(1-\beta_{n}\right) y_{n}, \quad n \geq 0 .
\end{array}\right.
$$

Suppose $\sum_{n=0}^{\infty}\left\|T\left(t_{n}\right) x_{n-1}-T\left(t_{n-1}\right) x_{n-1}\right\|<\infty$. Then $\left\{x_{n}\right\}$ converges strongly to $q$, as $n \rightarrow \infty$; where $q$ is the unique solution in $\operatorname{Fix}(\mathcal{S})$ to the variational inequality (2.2).

\section{Questions}

(i) Could we obtain Theorem 2.3 with other control conditions which are different from $(\mathrm{C} 2)$ and $(\mathrm{C} 3)$ ?

(ii) Could we weaken the control condition $\left(^{*}\right)$ by the strictly weaker condition $\left(^{* * *}\right)$ :

$$
\lim _{n \rightarrow \infty} \sup _{x \in \tilde{C}}\left\|T\left(t_{n}\right) x-T\left(t_{n-1}\right) x\right\|=0 ?
$$

The following theorem gives the affirmative answers to these question mentioned above.

Theorem 2.6. Let E be a reflexive Banach space which admits a weakly sequenctially continuous duality mapping $J$ from $E$ into $E^{*}$, suppose $C$ is a nonempty closed convex subset of $E$. Let $\{T(t): t \geq 0\}$, be a nonexpansive semigroup on $C$ such that $\operatorname{Fix}(\mathcal{S}) \neq \emptyset$, and $f: C \rightarrow C$ be a generalized contraction on $C$. Let $\left\{\alpha_{n}\right\} \subset(0,1),\left\{\beta_{n}\right\} \subset(0,1),\left\{\gamma_{n}\right\} \subset$ $[0,1]$ and $\left\{t_{n}\right\} \subset(0, \infty)$ be sequences of real numbers satisfying the conditions:

(B1) $\lim _{n \rightarrow \infty} \beta_{n}=0, \sum_{n=0}^{\infty} \beta_{n}=\infty$ and $\lim _{n \rightarrow \infty} t_{n}=0$,

(B2) $\alpha_{n}+\left(1+\alpha_{n}\right)\left(1-\gamma_{n}\right) \in[0$, a) for some $a \in(0,1)$,

(B3) $0<\lim \inf _{n \rightarrow \infty} \alpha_{n} \leq \lim \sup _{n \rightarrow \infty} \alpha_{n}<1$,

(B4) $\lim _{n \rightarrow \infty}\left(\gamma_{n+1}-\gamma_{n}\right)=0$. 
Define a sequence $\left\{x_{n}\right\}$ in $C$ by

$$
\left\{\begin{array}{l}
x_{0} \in C \\
z_{n}=\gamma_{n} x_{n}+\left(1-\gamma_{n}\right) T\left(t_{n}\right) x_{n} \\
y_{n}=\alpha_{n} x_{n}+\left(1-\alpha_{n}\right) T\left(t_{n}\right) z_{n} \\
x_{n+1}=\beta_{n} f\left(x_{n}\right)+\left(1-\beta_{n}\right) y_{n}, \quad n \geq 0 .
\end{array}\right.
$$

Suppose

$$
\lim _{n \rightarrow \infty} \sup _{x \in \tilde{C}}\left\|T\left(t_{n}\right) x-T\left(t_{n-1}\right) x\right\|=0,
$$

where $\tilde{C}$ is any bounded subset of $C$. Then $\left\{x_{n}\right\}$ converges strongly to $q$, as $n \rightarrow \infty$; where $q$ is the unique solution in $\operatorname{Fix}(\mathcal{S})$ to the variational inequality (2.2).

Proof. First, we show that $\left\{x_{n}\right\}$ is bounded. Indeed, if we take a fixed point $x \in \operatorname{Fix}(\mathcal{S})$. We will prove by induction that

$$
\left\|x_{n}-x\right\| \leq M \text { for all } n \geq 0,
$$

where $M:=\left\{|| x_{0}-z||, \eta^{-1}(|f f(x)-x| \mid)\right\}$. From Definition of (2.22), notice that

$$
\left\|z_{n}-x\right\| \leq \gamma_{n}\left\|x_{n}-x\right\|+\left(1-\gamma_{n}\right)\left\|T\left(t_{n}\right) x_{n}-x\right\| \leq\left\|x_{n}-x\right\| .
$$

It follows that

$$
\left\|y_{n}-x\right\| \leq \alpha_{n}\left\|x_{n}-x\right\|+\left(1-\alpha_{n}\right)\left\|T\left(t_{n}\right) z_{n}-x\right\| \leq \alpha_{n}\left\|x_{n}-x\right\|+\left(1-\alpha_{n}\right)\left\|x_{n}-x\right\| \leq\left\|x_{n}-x\right\| .
$$

The case $n=0$ is obvious.

Suppose that $\left\|x_{n}-x\right\| \leq M$, we have

$$
\begin{aligned}
\left\|x_{n+1}-x\right\| & \leq \beta_{n}\left\|f\left(x_{n}\right)-x\right\|+\left(1-\beta_{n}\right)\left\|y_{n}-x\right\| \\
& \leq \beta_{n}\left\|f\left(x_{n}\right)-f(x)\right\|+\beta_{n}\|f(x)-x\|+\left(1-\beta_{n}\right)\left\|y_{n}-x\right\| \\
& \leq \beta_{n} \psi\left(\left\|x_{n}-x\right\|\right)+\beta_{n}\|f(x)-x\|+\left(1-\beta_{n}\right)\left\|x_{n}-x\right\| \\
& =\beta_{n} \psi\left(\left\|x_{n}-x\right\|\right)+\beta_{n} \eta\left(\eta^{-1}(\|f(x)-x\|)\right)+\left(1-\beta_{n}\right)\left\|x_{n}-x\right\| \\
& \leq \beta_{n} \psi(M)+\beta_{n} \eta(M)+\left(1-\beta_{n}\right) M \\
& =\beta_{n} \psi(M)+\beta_{n}(M-\psi(M))+\left(1-\beta_{n}\right) M=M .
\end{aligned}
$$

By induction,

$$
\left\|x_{n}-x\right\| \leq \max \left\{\left\|x_{0}-x\right\|, \eta^{-1}(\|f(x)-x\|)\right\}, \quad \forall n \geq 0 .
$$

Thus $\left\{x_{n}\right\}$ is bounded, and so are $\left\{T\left(t_{n}\right) x_{n}\right\},\left\{y_{n}\right\},\left\{z_{n}\right\}$ and $\left\{f\left(x_{n}\right)\right\}$. As a result, we obtain by condition (B1),

$$
\left\|x_{n+1}-y_{n}\right\|=\beta_{n}\left\|f\left(x_{n}\right)-y_{n}\right\| \rightarrow 0 .
$$

We next show that

$$
\left\|x_{n}-T\left(t_{n}\right) x_{n}\right\| \rightarrow 0 .
$$

It suffices to show that

$$
\left\|x_{n+1}-x_{n}\right\| \rightarrow 0 \text {. }
$$


Indeed, if (2.25) holds, then noting (2.23), we obtain

$$
\begin{aligned}
\left\|x_{n}-T\left(t_{n}\right) x_{n}\right\| & \leq\left\|x_{n}-x_{n+1}\right\|+\left\|x_{n+1}-y_{n}\right\|+\left\|y_{n}-T\left(t_{n}\right) z_{n}\right\|+\left\|T\left(t_{n}\right) z_{n}-T\left(t_{n}\right) x_{n}\right\| \\
& \leq\left\|x_{n}-x_{n+1}\right\|+\left\|x_{n+1}-y_{n}\right\|+\alpha_{n}\left\|x_{n}-T\left(t_{n}\right) z_{n}\right\|+\left\|z_{n}-x_{n}\right\| \\
& \leq\left\|x_{n}-x_{n+1}\right\|+\left\|x_{n+1}-y_{n}\right\|+\alpha_{n}\left\|x_{n}-T\left(t_{n}\right) x_{n}\right\|+\alpha_{n}\left\|T\left(t_{n}\right) x_{n}-T\left(t_{n}\right) z_{n}\right\| \\
& +\left\|z_{n}-x_{n}\right\| \\
& \leq\left\|x_{n}-x_{n+1}\right\|+\left\|x_{n+1}-y_{n}\right\|+\alpha_{n}\left\|x_{n}-T\left(t_{n}\right) x_{n}\right\|+\alpha_{n}\left\|x_{n}-z_{n}\right\|+\left\|z_{n}-x_{n}\right\| \\
& \leq\left\|x_{n}-x_{n+1}\right\|+\left\|x_{n+1}-y_{n}\right\|+\alpha_{n}\left\|x_{n}-T\left(t_{n}\right) x_{n}\right\|+\left(1+\alpha_{n}\right)\left\|x_{n}-z_{n}\right\| \\
& \leq\left\|x_{n}-x_{n+1}\right\|+\left\|x_{n+1}-y_{n}\right\|+\alpha_{n}\left\|x_{n}-T\left(t_{n}\right) x_{n}\right\| \\
& +\left(1+\alpha_{n}\right)\left(1-\gamma_{n}\right)\left\|x_{n}-T\left(t_{n}\right) x_{n}\right\|
\end{aligned}
$$

and hence $\left(1-\left(\alpha_{n}+\left(1+\alpha_{n}\right)\left(1-\gamma_{n}\right)\right)\right)|| x_{n}-T\left(t_{n}\right) x_{n}|| \leq|| x_{n}-x_{n+1}||+|| x_{n+1}-y_{n} \| \rightarrow 0$ as $n \rightarrow \infty$. Using (B2), we conclude that (2.24) holds. Define the sequence $\left\{u_{n}\right\}$ by

$$
u_{n}=\frac{x_{n+1}-\sigma_{n} x_{n}}{\left(1-\sigma_{n}\right)}
$$

where $\sigma_{n}=\left(1-\beta_{n}\right) \alpha_{n}$. Then $x_{n+1}=\sigma_{n} x_{n}+\left(1-\sigma_{n}\right) u_{n}$. Next multiplication gives us that

$$
\begin{aligned}
u_{n+1}-u_{n}= & \frac{\left(x_{n+2}-\sigma_{n+1} x_{n+1}\right)}{1-\sigma_{n+1}}-\frac{x_{n+1}-\sigma_{n} x_{n}}{1-\sigma_{n}} \\
= & \frac{\beta_{n+1} f\left(x_{n+1}\right)+\left(1-\beta_{n+1}\right) y_{n+1}-\sigma_{n+1} x_{n+1}}{1-\sigma_{n+1}}-\frac{\beta_{n} f\left(x_{n}\right)+\left(1-\beta_{n}\right) y_{n}-\sigma_{n} x_{n}}{1-\sigma_{n}} \\
= & \left(\frac{\beta_{n+1} f\left(x_{n+1}\right)}{1-\alpha_{n+1}}-\frac{\beta_{n} f\left(x_{n}\right)}{1-\alpha_{n}}\right) \\
& +\frac{\left(1+\beta_{n+1}\right)\left(\alpha_{n+1} x_{n+1}+\left(1-\alpha_{n+1}\right) T\left(t_{n+1}\right) z_{n+1}\right)-\alpha_{n+1} x_{n+1}}{1-\sigma_{n+1}} \\
& -\frac{\left(1-\beta_{n}\right)\left(\alpha_{n} x_{n}+\left(1-\alpha_{n}\right) T\left(t_{n}\right) z_{n}\right)-\alpha_{n} x_{n}}{1-\sigma_{n}} \\
= & \left(\frac{\beta_{n+1} f\left(x_{n+1}\right)}{1-\sigma_{n+1}}-\frac{\beta_{n} f\left(x_{n}\right)}{1-\sigma_{n}}\right) \\
& +\left(T\left(t_{n+1}\right) z_{n+1}-\frac{\alpha_{n+1}}{1-\sigma_{n+1}} T\left(t_{n+1}\right) z_{n+1}\right)-\left(T\left(t_{n}\right) z_{n}-\frac{\alpha_{n}}{1-\sigma_{n}} T\left(t_{n}\right) z_{n}\right) \\
= & \frac{\beta_{n+1}}{1-\sigma_{n+1}}\left(f\left(x_{n+1}\right)-T\left(t_{n+1}\right) z_{n+1}\right)-\frac{\beta_{n}}{1-\sigma_{n}}\left(f\left(x_{n}\right)-T\left(t_{n}\right) z_{n}\right) \\
& +\left(T\left(t_{n+1}\right) z_{n+1}-T\left(t_{n+1}\right) z_{n}\right)-\left(T\left(t_{n+1}\right) z_{n}-T\left(t_{n}\right) z_{n}\right) .
\end{aligned}
$$

Then we have

$$
\begin{aligned}
\left\|u_{n+1}-u_{n}\right\| \leq & \frac{\beta_{n+1}}{1-\sigma_{n+1}}\left\|f\left(x_{n+1}\right)-T\left(t_{n+1}\right) z_{n+1}\right\|-\frac{\beta_{n}}{1-\sigma_{n}}\left\|f\left(x_{n}\right)-T\left(t_{n}\right) z_{n}\right\| \\
& +\left\|z_{n+1}-z_{n}\right\|+\left\|T\left(t_{n+1}\right) z_{n}-T\left(t_{n}\right) z_{n}\right\| .
\end{aligned}
$$

From (2.22) we have

$$
\begin{aligned}
z_{n+1}-z_{n}= & \gamma_{n+1} x_{n+1}+\left(1-\gamma_{n+1}\right) T\left(t_{n+1}\right) x_{n+1}-\gamma_{n} x_{n}-\left(1-\gamma_{n}\right) T\left(t_{n}\right) x_{n} \\
= & \gamma_{n+1}\left(x_{n+1}-x_{n}\right)+\left(\gamma_{n+1}-\gamma_{n}\right) x_{n}+\left(1-\gamma_{n+1}\right)\left(T\left(t_{n+1}\right) x_{n+1}-T\left(t_{n+1}\right) x_{n}\right) \\
& +\left(1-\gamma_{n+1}\right)\left(T\left(t_{n+1}\right) x_{n}-T\left(t_{n}\right) x_{n}\right)-\left(\gamma_{n+1}-\gamma_{n}\right) T\left(t_{n}\right) x_{n} \\
= & \gamma_{n+1}\left(x_{n+1}-x_{n}\right)+\left(\gamma_{n+1}-\gamma_{n}\right)\left(x_{n}-T\left(t_{n}\right) x_{n}\right) \\
& +\left(1-\gamma_{n+1}\right)\left(T\left(t_{n+1}\right) x_{n+1}-T\left(t_{n+1}\right) x_{n}\right)+\left(1-\gamma_{n+1}\right)\left(T\left(t_{n+1}\right) x_{n}-T\left(t_{n}\right) x_{n}\right),
\end{aligned}
$$


that is,

$$
\begin{aligned}
\left\|z_{n+1}-z_{n}\right\| \leq & \gamma_{n+1}\left\|x_{n+1}-x_{n}\right\|+\left|\gamma_{n+1}-\gamma_{n}\right|\left\|x_{n}-T\left(t_{n}\right) x_{n}\right\|+\left(1-\gamma_{n+1}\right)\left\|x_{n+1}-x_{n}\right\| \\
& +\left(1-\gamma_{n+1}\right)\left\|T\left(t_{n+1}\right) x_{n}-T\left(t_{n}\right) x_{n}\right\| \\
\leq & \left\|x_{n+1}-x_{n}\right\|+\left|\gamma_{n+1}-\gamma_{n}\right|\left\|x_{n}-T\left(t_{n}\right) x_{n}\right\| T\left(t_{n+1}\right) x_{n}-T\left(t_{n}\right) x_{n} \| .
\end{aligned}
$$

Substituting (2.27) into (2.26) that

$$
\begin{aligned}
\left\|u_{n+1}-u_{n}\right\|-\left\|x_{n+1}-x_{n}\right\| \leq & \frac{\beta_{n+1}}{1-\sigma_{n+1}}\left\|f\left(x_{n+1}\right)-T\left(t_{n+1}\right) z_{n+1}\right\|-\frac{\beta_{n}}{1-\sigma_{n}}\left\|f\left(x_{n}\right)-T\left(t_{n}\right) z_{n}\right\| \\
& +\left|\gamma_{n+1}-\gamma_{n}\right|\left\|x_{n}-T\left(t_{n}\right) x_{n}\right\|+\left\|T\left(t_{n+1}\right) x_{n}-T\left(t_{n}\right) x_{n}\right\| \\
& +\left\|T\left(t_{n+1}\right) z_{n}-T\left(t_{n}\right) z_{n}\right\| \\
\leq & \frac{\beta_{n+1}}{1-\sigma_{n+1}}\left\|f\left(x_{n+1}\right)-T\left(t_{n+1}\right) z_{n+1}\right\|-\frac{\beta_{n}}{1-\sigma_{n}}\left\|f\left(x_{n}\right)-T\left(t_{n}\right) z_{n}\right\| \\
& +\left|\gamma_{n+1}-\gamma_{n}\right|\left\|x_{n}-T\left(t_{n}\right) x_{n}\right\|+\sup _{x \in\left\{x_{n}\right\}}\left\|T\left(t_{n+1}\right) x-T\left(t_{n}\right) x\right\| \\
& +\sup _{z \in\left\{z_{n}\right\}}\left\|T\left(t_{n+1}\right) z-T\left(t_{n}\right) z\right\| .
\end{aligned}
$$

By (B1),

(B4), $\lim _{n \rightarrow \infty} \sup _{x \in\left\{x_{n}\right\}}\left\|T\left(t_{n+1}\right) x-T\left(t_{n}\right) x\right\|=0$,

$\lim _{n \rightarrow \infty} \sup _{z \in\left\{z_{n}\right\}}\left\|T\left(t_{n+1}\right) z-T\left(t_{n}\right) z\right\|=0$ and (2.28), we obtain that

$$
\limsup _{n \rightarrow \infty}\left(\left\|u_{n+1}-u_{n}\right\|-\left\|x_{n+1}-x_{n}\right\|\right) \leq 0 .
$$

Hence by Lemma 1.8, we have $\lim _{n \rightarrow \infty}|| u_{n}-x_{n} \|=0$. It follows from (B3) that

$$
\lim _{n \rightarrow \infty}\left\|x_{n+1}-x_{n}\right\|=\lim _{n \rightarrow \infty}\left(1-\sigma_{n}\right)\left\|u_{n}-x_{n}\right\|=0 .
$$

Hence (2.24) holds. Applying Theorem 2.1, there is a unique solution $q \in \operatorname{Fix}(\mathcal{S})$ to the following variational inequality:

$$
\langle f(q)-q, j(x-q)\rangle \leq 0 \quad \text { for all } x \in \operatorname{Fix}(\mathcal{S}) .
$$

Next, we show that

$$
\limsup _{n \rightarrow \infty}\left\langle f(q)-q, j\left(x_{n+1}-q\right)\right\rangle \leq 0 .
$$

Indeed, we can take a subsequence $\left\{x_{n_{i}}\right\}$ of $\left\{x_{n}\right\}$ such that

$$
\limsup _{n \rightarrow \infty}\left\langle f(q)-q, j\left(x_{n+1}-q\right)\right\rangle=\lim _{i \rightarrow \infty}\left\langle f(q)-q, j\left(x_{n+1}-q\right)\right\rangle .
$$

By the reflexivity of $E$ and boundedness of the sequence $\left\{x_{n}\right\}$, we may assume, without loss of generality, that $x_{n_{i}} \rightarrow p$ for some $p \in C$. Now we show that $p \in \operatorname{Fix}(\mathcal{S})$. Put $x_{i}=x_{n_{i}}, \alpha_{i}=\alpha_{n_{i}}, \beta_{i}=\beta_{n_{i}}$ and $t_{i}=t_{n_{i}}$ for $i \in \mathbb{N}$, let $t_{i} \geq 0$ be such that

$$
t_{i} \rightarrow 0 \text { and } \frac{\left\|T\left(t_{i}\right) x_{i}-x_{i}\right\|}{t_{i}} \rightarrow 0, \quad i \rightarrow \infty .
$$

Fix $t>0$. Notice that

$$
\begin{aligned}
\left\|x_{i}-T(t) p\right\| \leq & \sum_{k=0}^{\left[t / t_{i}\right]-1}\left\|T\left((k+1) t_{i}\right) x_{i}-T\left(k t_{i}\right) x_{i}\right\| \\
& +\left\|T\left(\left[t / t_{i}\right] t_{i}\right) x_{i}-T\left(\left[t / t_{i}\right] t_{i}\right) p\right\|+\left\|T\left(\left[t / t_{i}\right] t_{i}\right) p-T(t) p\right\| \\
\leq & {\left[t / t_{i}\right]\left\|T\left(t_{i}\right) x_{i}-x_{i}\right\|+\left\|x_{i}-p\right\|+\left\|T\left(t-\left[t / t_{i}\right] t_{i}\right) p-p\right\| } \\
\leq & t \frac{\left\|T\left(t_{i}\right) x_{i}-x_{i}\right\|}{t_{i}}+\left\|x_{i}-p\right\|+\left\|T\left(t-\left[t / t_{i}\right] t_{i}\right) p-p\right\| \\
& \leq t \frac{\left\|T\left(t_{i}\right) x_{i}-x_{i}\right\|}{t_{i}}+\left\|x_{i}-p\right\|+\max \left\{\|T(s) p-p\|: 0 \leq s \leq t_{i}\right\} .
\end{aligned}
$$


For all $i \in \mathbb{N}$, we have

$$
\limsup _{i \rightarrow \infty}\left\|x_{i}-T(t) p\right\| \leq \limsup _{i \rightarrow \infty}\left\|x_{i}-p\right\| .
$$

Since Banach space $E$ with a weakly sequentially continuous duality mapping satisfies Opial's condition, this implies $T(t) p=p$. Therefore $p \in \operatorname{Fix}(\mathcal{S})$. In view of the variational inequality (2.2) and the assumption that duality mapping $J$ is weakly sequentially continuous, we conclude

$$
\begin{aligned}
\limsup _{n \rightarrow \infty}\left\langle f(q)-q_{,} j\left(x_{n+1}-q\right)\right\rangle & =\lim _{i \rightarrow \infty}\left\langle f(q)-q, j\left(x_{n i+1}-q\right)\right\rangle \\
& =\langle f(q)-q, j(p-q)\rangle \leq 0 .
\end{aligned}
$$

Then (2.29) is proved. Finally, show that $x_{n} \rightarrow q$, i.e., $\left\|x_{n}-q\right\| \rightarrow 0$. Suppose that $\| x_{n^{-}}$ $q \| \nrightarrow 0$, then there exists $\varepsilon>0$ and a subsequence $\left\{x_{n_{j}}\right\}$ of $\left\{x_{n}\right\}$ such that $\left\|x_{n_{j}}-q\right\| \geq \varepsilon$ for all $j \in \mathbb{N}$. Put $x_{j}=x_{n_{j}}, \alpha_{j}=\alpha_{n_{j}}, \beta_{j}=\beta_{n_{j}}$ and $t_{j}=t_{n_{j}}$ for $j \in \mathbb{N}$. By Proposition 1.4, there exists $r \in(0,1)$ such that $\left\|f\left(x_{j}\right)-f(q)\right\| \leq r\left\|x_{j}-q\right\|$ for all $j \in \mathbb{N}$. As a matter of fact, from 1.6 we have that

$$
\begin{aligned}
\left\|x_{j+1}-q\right\|^{2}= & \left\|\beta_{j} f\left(x_{j}\right)+\left(1-\beta_{j}\right)\left(\alpha_{j} x_{j}+\left(1-\alpha_{j}\right) T\left(t_{j}\right) z_{j}\right)-q\right\|^{2} \\
= & \|\left(1-\beta_{j}\right)\left(\alpha_{j}\left(x_{j}-q\right)+\left(1-\alpha_{j}\right)\left(T\left(t_{j} z_{j}-q\right)\right)+\beta_{j}\left(f\left(x_{j}\right)-q\right) \|^{2}\right. \\
\leq & \left(1-\beta_{j}\right)^{2}\left\|\alpha_{j}\left(x_{j}-q\right)+\left(1-\alpha_{j}\right)\left(T\left(t_{j}\right) z_{j}-q\right)\right\|^{2}+2 \beta_{j}\left\langle f\left(x_{j}\right)-q, j\left(x_{j+1}-q\right)\right\rangle \\
\leq & \left(1-\beta_{j}\right)^{2}\left(\alpha_{j}\left\|x_{j}-q\right\|+\left(1-\alpha_{j}\right)\left\|T\left(t_{j}\right) z_{j}-q\right\|\right)^{2} \\
& +2 \beta_{j}\left(f\left(x_{j}\right)-f(q), j\left(x_{j+1}-q\right)\right\rangle+2 \beta_{j}\left(f(q)-q, j\left(x_{j+1}-q\right)\right\rangle \\
\leq & \left(1-\beta_{j}\right)^{2}\left\|x_{j}-q\right\|^{2}+2 \beta_{j}\left\|f\left(x_{j}\right)-f(q)\right\|\left\|x_{j+1}-q\right\|+2 \beta_{j}\left\langle f(q)-q, j\left(x_{j+1}-q\right)\right\rangle \\
\leq & \left(1-\beta_{j}\right)^{2}\left\|x_{j}-q\right\|^{2}+2 \beta_{j} r\left\|x_{j}-q\right\|\left\|x_{j+1}-q\right\|+2 \beta_{j}\left\langle f(q)-q, j\left(x_{j+1}-q\right)\right\rangle \\
\leq & \left(1-\beta_{j}\right)^{2}\left\|x_{j}-q\right\|^{2}+\beta_{j} r\left(\left\|x_{j}-q\right\|^{2}+\left\|x_{j+1}-q\right\|^{2}\right)+2 \beta_{j}\left\langle f(q)-q, j\left(x_{j+1}-q\right)\right\rangle \\
\leq & \left(\left(1-\beta_{j}\right)^{2}+\beta_{j} r\right)\left\|x_{j}-q\right\|^{2}+\beta_{j} r\left\|x_{j+1}-q\right\|^{2}+2 \beta_{j}\left\langle f(q)-q, j\left(x_{j+1}-q\right)\right\rangle .
\end{aligned}
$$

It follows that

$$
\begin{aligned}
\left\|x_{j+1}-q\right\|^{2} & \leq \frac{\left(1-(2-r) \beta_{j}+\beta_{j}^{2}\right)}{1-\beta_{j} r}\left\|x_{j}-q\right\|^{2}+\frac{2 \beta_{j}}{1-\beta_{j} r}\left\langle f(q)-q, j\left(x_{j+1}-q\right)\right\rangle \\
& =\frac{1-(2-r) \beta_{j}}{1-\beta_{j} r}\left\|x_{j}-q\right\|^{2}+\frac{\beta_{j}^{2}}{1-\beta_{j} r}\left\|x_{j}-q\right\|^{2}+\frac{2 \beta_{j}}{1-\beta_{j} r}\left\langle f(q)-q, j\left(x_{j+1}-q\right)\right\rangle \\
& \leq \frac{1-(2-r) \beta_{j}}{1-\beta_{j} r}\left\|x_{j}-q\right\|^{2}+\frac{\beta_{j}^{2}}{1-r}\left\|x_{j}-q\right\|^{2}+\frac{2 \beta_{j}}{1-r}\left\langle f(q)-q, j\left(x_{j+1}-q\right)\right\rangle \\
& \leq \frac{1-\beta_{j} r-2(1-r) \beta_{j}}{1-\beta_{j} r}\left\|x_{j}-q\right\|^{2}+\beta_{j}^{2} M+\frac{2 \beta_{j}}{1-r}\left\langle f(q)-q, j\left(x_{j+1}-q\right)\right\rangle \\
& =\left(1-\frac{2(1-r) \beta_{j}}{1-\beta_{j} r}\right)\left\|x_{j}-q\right\|^{2}+\beta_{j}^{2} M+\frac{2 \beta_{j}}{1-r}\left\langle f(q)-q, j\left(x_{j+1}-q\right)\right\rangle \\
& \leq\left(1-2(1-r) \beta_{j}\right)\left\|x_{j}-q\right\|^{2}+\beta_{j}\left(\frac{2}{1-r}\left\langle f(q)-q, j\left(x_{j+1}-q\right)\right\rangle+\beta_{j} M\right),
\end{aligned}
$$

where $M>0$ such that $M \geq \frac{1}{1-r}\left\|x_{j}-q\right\|^{2}$. That is,

$$
\left\|x_{j+1}-q\right\|^{2} \leq\left(1-\gamma_{j}\right)\left\|x_{j}-q\right\|^{2}+\delta_{j},
$$

Where $\gamma_{j}=2(1-r) \beta_{j}$ and $\frac{\delta_{j}}{\gamma_{j}}=\frac{1}{(1-r)^{2}}\left\langle f(q)-q, j\left(x_{j+1}-q\right)\right\rangle+\frac{M}{2(1-r)} \beta_{j}$. 
It follows by condition (B1) that $\gamma_{j} \rightarrow 0$ and $\sum_{j=1}^{\infty} \gamma_{j}=\infty$. From (2.29) we have

$$
\begin{aligned}
\limsup _{j \rightarrow \infty} \frac{\delta_{j}}{\gamma_{j}} & \leq \limsup _{j \rightarrow \infty} \frac{1}{(1-r)^{2}}\left\langle f(q)-q, j\left(x_{j+1}-q\right)\right\rangle+\lim _{j \rightarrow \infty} \frac{M}{2(1-r)} \beta_{j} \\
& \leq \limsup _{j \rightarrow \infty} \frac{1}{(1-r)^{2}}\left\langle f(q)-q, j\left(x_{j+1}-q\right)\right\rangle \leq 0 .
\end{aligned}
$$

Using Lemma 1.7 onto (2.30), we conclude that $\left\|x_{j}-q\right\| \rightarrow 0$. This is a contradiction. Hence $x_{n} \rightarrow q$.

The proof is completed.

If $\gamma_{n} \equiv 1$, then we have the following Corollary.

Corollary 2.7. Let E be a reflexive Banach space which admits a weakly sequenctially continuous duality mapping $J$ from $E$ into $E^{*}$, suppose $C$ is a nonempty closed convex subset of $E$. Let $\{T(t): t \geq 0\}$, be a nonexpansive semigroup on $C$ such that $\operatorname{Fix}(\mathcal{S}) \neq \emptyset$, and $f: C \rightarrow C$ be a generalized contraction on $C$. Let $\left\{\alpha_{n}\right\} \subset(0,1),\left\{\beta_{n}\right\} \subset(0,1)$, and $\left\{t_{n}\right\}$ $\subset(0, \infty)$ be sequences of real numbers satisfying the conditions:

(B1) $\lim _{n \rightarrow \infty} \beta_{n}=0, \sum_{n=0}^{\infty} \beta_{n}=\infty$,

(B2) $0<\lim \inf _{n \rightarrow \infty} \alpha_{n} \leq \lim \sup _{n \rightarrow \infty} \alpha_{n}<1$,

(B3) $\lim _{n \rightarrow \infty} t_{n}=0$.

Define a sequence $\left\{x_{n}\right\}$ in $C$ by

$$
\left\{\begin{array}{l}
x_{0} \in C \\
y_{n}=\alpha_{n} x_{n}+\left(1-\alpha_{n}\right) T\left(t_{n}\right) x_{n} \\
x_{n+1}=\beta_{n} f\left(x_{n}\right)+\left(1-\beta_{n}\right) y_{n}, \quad n \geq 0 .
\end{array}\right.
$$

Suppose $\lim _{n \rightarrow \infty}\left\|T\left(t_{n}\right) x_{n-1}-T\left(t_{n-1}\right) x_{n-1}\right\|=0$. Then $\left\{x_{n}\right\}$ converges strongly to $q$, as $n$ $\rightarrow \infty$ where $q$ is the unique solution in $\operatorname{Fix}(\mathcal{S})$ to the variational inequality (2.2).

Setting $f$ is a contraction on $C$ in Corollary 2.7, we have the following results immediately.

Corollary 2.8. Let E be a reflexive Banach space which admits a weakly sequenctially continuous duality mapping J from $E$ into $E^{*}$, suppose $C$ is a nonempty closed convex subset of $E$. Let $\{T(t): t \geq 0\}$, be a nonexpansive semigroup on $C$ such that $\operatorname{Fix}(\mathcal{S}) \neq \emptyset$, and $f: C \rightarrow C$ be a contraction on $C$. Let $\left\{\alpha_{n}\right\} \subset(0,1),\left\{\beta_{n}\right\} \subset(0,1)$, and $\left\{t_{n}\right\} \subset(0, \infty)$ be sequences of real numbers satisfying the conditions:

(B1) $\lim _{n \rightarrow \infty} \beta_{n}=0, \sum_{n=0}^{\infty} \beta_{n}=\infty$,

(B2) $0<\liminf _{n \rightarrow \infty} \alpha_{n} \leq \lim \sup _{n \rightarrow \infty} \alpha_{n}<1$,

(B3) $\lim _{n \rightarrow \infty} t_{n}=0$.

Define a sequence $\left\{x_{n}\right\}$ in $C$ by

$$
\left\{\begin{array}{l}
x_{0} \in C \\
y_{n}=\alpha_{n} x_{n}+\left(1-\alpha_{n}\right) T\left(t_{n}\right) x_{n} \\
x_{n+1}=\beta_{n} f\left(x_{n}\right)+\left(1-\beta_{n}\right) y_{n}, \quad n \geq 0 .
\end{array}\right.
$$

Suppose $\lim _{n \rightarrow \infty}\left\|T\left(t_{n}\right) x_{n-1}-T\left(t_{n-1}\right) x_{n-1}\right\|=0$. Then $\left\{x_{n}\right\}$ converges strongly to $q$, as $n$ $\rightarrow \infty$ where $q$ is the unique solution in $\operatorname{Fix}(\mathcal{S})$ to the variational inequality (2.2). 
Remark 2.9. Theorem 2.3 generalize and improve [11, Theorem 3.2]. In fact,

(i) The iterations (1.10) can reduce to (1.9).

(ii) The contraction is replaced by the generalized contraction in both modified Mann iterations (1.8) and (1.9).

(iii) We can obtain the Theorem 2.3 with control conditions (B2), (B3), and (B4) which are different from $(\mathrm{C} 2)$ and $(\mathrm{C} 3)$.

\section{Acknowledgements}

The project was supported by the "Centre of Excellence in Mathematics" under the Commission on Higher Education, Ministry of Education, Thailand.

\section{Author details}

${ }^{1}$ Department of Mathematics, Faculty of Science, Naresuan University, Phitsanulok 65000, Thailand ${ }^{2}$ Centre of Excellence in Mathematics, CHE, Si Ayutthaya Road, Bangkok 10400, Thailand

\section{Authors' contributions}

All authors read and approved the final manuscript.

\section{Competing interests}

The authors declare that they have no competing interests.

Received: 1 June 2011 Accepted: 12 January 2012 Published: 12 January 2012

\section{References}

1. Lim, TC: On Characterizations of Mier-Keeler contractive maps. Nonlinear Anal. 46, 113-120 (2001). doi:10.1016/S0362546X(99)00448-4

2. Petrusel, A, Yao, JC: Viscosity approximation to common fixed points of nonexpansive mappings with generalized contractions mappings. Nonlinear Anal. 69, 1100-1111 (2008). doi:10.1016/j.na.2007.06.016

3. Suzuki, T: Moudafi's viscosity approximations with Mier-Keeler contractions. J Math Anal Appl. 325, 342-352 (2007). doi:10.1016/j.jmaa.2006.01.080

4. Shioji, N, Takahashi, W: Strong convergence theorems for asymptotically nonexpansive semigroups in Hilbert spaces. Nonlinear Anal. 34, 87-99 (1998). doi:10.1016/S0362-546X(97)00682-2

5. Shimizu, T, Takahashi, W: Strong convergence to common fixed points of families of nonexpansive mappings. J Math Anal Appl. 211, 71-83 (1997). doi:10.1006/jmaa.1997.5398

6. Chen, R, Song, Y: Convergence to common fixed point of nonexpansive semigroups. J Comput Appl Math. 200, 566-575 (2007). doi:10.1016/j.cam.2006.01.009

7. Suzuki, T: On strong convergence to common fixed points of nonexpansive semigroups in Hilbert spaces. Proc Am Math Soc. 131, 2133-2136 (2003). doi:10.1090/S0002-9939-02-06844-2

8. Benavides, TD, Acedo, GL, Xu, HK: Construction of sunny nonexpansive retractions in Banach spaces. Bull Aust Math Soc. 66(1), 9-16 (2002). doi:10.1017/S0004972700020621

9. Xu, HK: A strong convergence theorem for contraction semigroups in Banach spaces. Bull Aust Math Soc. 72, 371-379 (2005). doi:10.1017/S000497270003519X

10. Chen, $\mathrm{R}, \mathrm{He}, \mathrm{H}$ : Viscosity approximation of common fixed points of nonexpansive semigroups in Banach space. Appl Math Lett. 20, 751-757 (2007). doi:10.1016/j.aml.2006.09.003

11. Chen, RD, He, HM, Noor, MA: Modified Mann iterations for nonexpansive semigroups in Banach space. Acta Math Sin. 26(1), 193-202 (2010). doi:10.1007/s10114-010-7446-7

12. Moudafi, A: Viscosity approximation methods for fixed-points problems. J Math Anal Appl. 241, 46-55 (2000). doi:10.1006/jmaa.1999.6615

13. Xu, HK: Viscosity approximation methods for nonexpansive mappings. J Math Anal Appl. 298, 279-291 (2004) doi:10.1016/j.jmaa.2004.04.059

14. Suzuki, T: Moudafi's viscosity approximations with Meir-Keeler contractions. J Math Anal Appl. 325, 342-352 (2007). doi:10.1016/j.jmaa.2006.01.080

15. Xu, B, Noor, MA: Fixed-point iterations for asymptotically nonexpansive mappings in Banach spaces. J Math Anal Appl. 267, 444-453 (2002). doi:10.1006/jmaa.2001.7649

16. Noor, MA: New approximation schemes for general variational inequalities. J Math Anal Appl. 251, $217-229$ (2000). doi:10.1006/jmaa.2000.7042

17. Noor, MA: Some developments in general variational inequalities. Appl Math Comput. 152, 199-277 (2004). doi:10.1016/ S0096-3003(03)00558-7

18. Noor, MA: Extended general variational inequalities. Appl Math Lett. 22, 182-185 (2009). doi:10.1016/j.aml.2008.03.007

19. Noor, MA: Three-step iterative algorithms for multivalued quasi variational inclusions. J Math Anal Appl. 225, 589-604 (2001)

20. Yao, Y, Noor, MA: Convergence of three-step iterations for asymptotically nonexpansive mappings. Comput Math Appl. 187, 883-892 (2007). doi:10.1016/j.amc.2006.09.008

21. Plubtieng, S, Wangkeeree, R: Noor Iterations with error for non-lipschitzian mappings in Banach spaces. KYUNG-POOK Math J. 46, 201-209 (2006) 
22. Plubtieng, S, Wangkeeree, R, Punpaeng, R: On the convergence of modified Noor iterations with errors for asymptotically nonexpansive mappings. J Math Anal Appl. 322, 1018-1029 (2006). doi:10.1016/j.jmaa.2005.09.078

23. Plubtieng, S, Wangkeeree, R: Strong convergence theorems for multi-step Noor iterations with errors in Banach spaces. J Math Anal Appl. 321, 10-23 (2006). doi:10.1016/j.jmaa.2005.08.029

24. Browder, FE: Convergence of approximants to fixed points of nonexpansive non-linear mappings in Banach spaces. Arch Ration Mech Anal. 24, 82-90 (1967)

25. Gossez, JP, Dozo, EL: Some geometric Properties related to the fixed point theory for nonexpansive mappings. Pac J Math. 40, 565-573 (1972)

26. Xu, HK: An iterative approach to quadratic optimization. J Optim Theory Appl. 116, 659-678 (2003). doi:10.1023/ A:1023073621589

27. Suzuki, T: Strong convergence of approximated sequences for nonexpansive mappings in Banach spaces. Proc Am Math Soc. 135, 99-106 (2007)

doi:10.1186/1029-242X-2012-6

Cite this article as: Wangkeeree and Preechasilp: Modified noor iterations for nonexpansive semigroups with generalized contraction in Banach spaces. Journal of Inequalities and Applications 2012 2012:6.

\section{Submit your manuscript to a SpringerOpen ${ }^{\circ}$} journal and benefit from:

- Convenient online submission

- Rigorous peer review

- Immediate publication on acceptance

- Open access: articles freely available online

- High visibility within the field

- Retaining the copyright to your article

Submit your next manuscript at $\boldsymbol{\nabla}$ springeropen.com 